\title{
Electrical and thermal properties of a two-dimensional electron gas in a one-dimensional periodic potential
}

\author{
F. M. Peeters \\ Department of Physics, University of Antwerp (UIA), B-2610 Antwerp, Belgium \\ P. Vasilopoulos \\ Department of Physics, Concordia University, Montréal, Canada H3G 1 M8
}

(Received 7 December 1990; revised manuscript received 21 May 1991)

\begin{abstract}
We investigate the influence of a periodic weak modulation along the $x$ direction on the electrical and thermal properties of a two-dimensional electron gas in the presence of a perpendicular magnetic field. The modulation lifts the degeneracy of the Landau levels and leads to one-dimensional magnetic bands whose bandwidth oscillates as a function of the magnetic field. At weak magnetic fields this gives rise to the Weiss oscillations in the magnetoresistance, discovered recently, which have a very weakly temperature-dependent amplitude and a period proportional to $\sqrt{n_{e}}$, when $n_{e}$ is the electron density. Diffusion-current contributions, proportional to the square of the bandwidth, dominate $\rho_{x x}$, and collisional contributions, varying approximately as the square of the density of states, dominate $\rho_{y y}$. The result is that $\rho_{x x}$ and $\rho_{y y}$ oscillate out of phase as observed. Asymptotic analytical expressions are presented for the conductivity tensor. Similar oscillations, of much smaller amplitude, occur in the thermodynamic quantities, such as the magnetization, the susceptibility, and the specific heat. We also predict oscillations in the Hall resistance, the cyclotron resonance position, the linewidth, as well as in the thermal conductivity and thermopower. The components of the thermal-resistance tensor have a magnetic-field dependence similar to that of the electrical-resistivity tensor.
\end{abstract}

\section{INTRODUCTION}

A new type of magnetoresistance oscillation (hereafter called Weiss oscillations) in a two-dimensional electron gas (2DEG) modulated by a lateral periodic electric potential has been observed recently. ${ }^{1-3}$ Experimentally, two different techniques have been used to create the 1D modulation: (1) Weiss et al. ${ }^{1}$ used a holographic technique to create, by illumination, a pattern of parallel fringes as produced by two interfering laser beams. The light pulses ionize $D X$ centers in the $\mathrm{Al}_{1-x} \mathrm{Ga}_{x}$ As layer by means of the persistent photoconductivity effect. The resulting stripe-shaped areas of ionized impurities modulate the electrostatic potential of the adjacent 2DEG; (2) Winkler, Kotthaus, and $\mathrm{Ploog}^{3}$ produced a 1D superlattice by depositing an array of parallel metallic strips on the surface of the $\mathrm{Al}_{1-x} \mathrm{Ga}_{x}$ As layer. By biasing these stripes, an electrostatically induced 1D potential is created. Recently, this work was extended to a 2D modulation with a square geometry ${ }^{4-6}$ and to a hexagonal geometry $^{7}$ with the aim to observe the Hofstadter lattice. ${ }^{8}$

Such a weak one-dimensional periodic modulation in the $x$ direction introduces a length scale in the problem: the period of the modulation $a$. The 2DEG is subjected to a perpendicular magnetic field $B=B e_{z}$ with a natural length scale: the cyclotron radius at the Fermi energy $R_{c}=\sqrt{2 \pi n_{e}} l^{2}$, where $l=\sqrt{\hbar c / e B}$ is the magnetic length. The oscillations in the magnetoresistance $\rho_{\mu v}$ are a consequence of the commensurability between those two length scales and have the following characteristics: (1) they are periodic in $1 / B$ like the Shubnikov-de Haas
(SdH) oscillations; (2) their period varies with electron density $\left(n_{e}\right)$ as $\sqrt{n_{e}}$, whereas that of the $\mathrm{SdH}$ ones as $n_{e}$; (3) their amplitude depends on the temperature much less than that of the SdH oscillations; (4) they are visible at weak magnetic fields, typically $B<0.4 \mathrm{~T}$, and at higher fields the $\mathrm{SdH}$ oscillations are modulated by these oscillations; (5) $\rho_{x x}$ and $\rho_{y y}$ oscillate out of phase and the magnitude of these oscillations $\left|\Delta \rho_{\mu v}\right|$ is such that $\left|\Delta \rho_{y y}\right| \ll\left|\Delta \rho_{x x}\right|$.

At present, several theoretical calculations s, $^{2,9-11}$ exist which are able to explain the oscillations in the magnetoresistance component $\rho_{x x}$ perpendicular to the periodic potential. Beenakker ${ }^{9}$ showed that these oscillations can be understood as being a semiclassical effect: they are due to guiding-center-drift resonances. The resistivity component along the periodic $\rho_{y y}$ shows weak oscillations which are an order of magnitude smaller than those in $\rho_{x x}$, and they were not explained in the early theoretical treatments. ${ }^{2,3,9}$ They have a quantum-mechanical origin and were explained by us ${ }^{10}$ and by Zhang and Gerhardts. $^{11}$ In Ref. 11 a self-consistent theory was presented, while we showed that such a complicated theory is not necessary in order to explain the basic physics which is responsible for the oscillations in $\rho_{y y}$. Středa and MacDonald ${ }^{12}$ used the idea of magnetic breakdown to explain the positive magnetoresistance in $\rho_{x x}$ and found that the oscillations are a consequence of oscillations of the breakdown transition probability. No attempt was made in Ref. 12 to explain the oscillations in $\rho_{y y}$.

The aim of the present paper is threefold. First of all, 
we want to give the details of our calculation which are missing in Ref. 10. Second, explicit analytic expressions are derived for the components of the resistivity tensor which are valid when many Landau levels are occupied. These expressions are able to reproduce the Weiss and $\mathrm{SdH}$ oscillations and reduce to the known results in the absence of modulation. Third, we present a detailed investigation of the thermodynamics of such a modulated 2DEG and calculate also the thermal transport coefficients like the thermal conductivity and thermopower. A brief account of the latter was presented in Ref. 13.

The paper is organized as follows. In Sec. II we calculate the energy spectrum and the density of states (DOS). A simple classical picture is given to illustrate the removal of the degeneracy of the Landau levels. The thermodynamic quantities such as the magnetization, the susceptibility, and the specific heat are calculated in Sec. III. Next, in Sec. IV, the electrical conductivity and resistivity tensors are calculated. Also, oscillations in the Hall resistance are predicted which show up in a more pronounced fashion in its derivative $d R_{H} / d B$. Analytic expressions for the resistivity components $\rho_{x x}$ and $\rho_{y y}$ are presented which are valid in the asymptotic limit of many occupied Landau levels. The calculation of the thermal transport coefficients is presented in Sec. V, and of the cyclotron resonance absorption spectrum in Sec. VI. Finally our conclusions are given in Sec. VII.

\section{ENERGY SPECTRUM}

We consider a $2 \mathrm{DEG}$, in the $x, y$ plane, subject to a magnetic field $\mathbf{B}$ along the $z$ direction, and a 1D weak periodic modulation $U(x)$ along the $x$ direction. The one-electron Hamiltonian reads

$$
H=\frac{1}{2 m^{*}}\left[\mathbf{p}+\frac{e}{c} \mathbf{A}\right]^{2}+U(x),
$$

where $\mathbf{p}$ is the momentum operator and $m^{*}$ the effective mass. In the absence of the modulation, i.e., for $U(x)=0$, and for the vector potential chosen in the Landau gauge $\mathbf{A}=(0, B x, 0)$, the normalized eigenfunctions of Eq. (1) are given by $\Phi_{n}\left(x+x_{0}\right) \exp \left(i y k_{y}\right) / \sqrt{L_{y}}$, where $\Phi_{n}(x)$ are the harmonic-oscillator wave functions centered at $x_{0}=l^{2} k_{y}$ with $n$ being the Landau-level index, $l=\sqrt{\hbar c / e B}$ the magnetic length, and $L_{y}$ the length of the $2 \mathrm{DEG}$ in the $y$ direction. The corresponding eigenvalue is $E_{n}=(n+1 / 2) \hbar \omega_{c}$, which is degenerate with respect to the wave vector $k_{y}\left(\omega_{c}=e B / m^{*} c\right.$ is the cyclotron frequency).

The modulation potential is usually approximated by the first Fourier component of the periodic potential, i.e., $U(x)=V_{0} \cos (2 \pi x / a)$, which is expected to be a good approximation in particular for the electrostatically induced 1D periodic potentials. In the presence of the modulation $U(x)$ the exact eigenstates of Eq. (1) are difficult to obtain in closed analytical form. In the reported experiments the amplitude of the modulation is small. Therefore, we can evaluate the correction to the energy levels by first-order perturbation theory using the unperturbed wave functions given above. In Ref. 2 an exact diagonalization of Eq. (1) showed that this was an excellent approximation for $n>4$, while in the experiments the relevant $n$ is about 10 . For $U(x)$ $=V_{0} \cos (K x), K=2 \pi / a$, we obtain

$$
E_{n, k_{y}}=\left(n+\frac{1}{2}\right) \hbar \omega_{c}+V_{0} \cos \left(K x_{0}\right) e^{-u / 2} L_{n}(u),
$$

where $u=K^{2} l^{2} / 2$ and $L_{n}(u)$ is a Laguerre polynomial. Since $x_{0}=l^{2} k_{y}$ we see that the presence of the modulation lifts the $k_{y}$ degeneracy: the electron energy depends on the position of the center of the cyclotron orbit $x_{0}$. This result should be valid as long as the separation between the Landau levels is larger than the broadening of the levels. For completeness we mention that the energy spectrum of such a one-dimensional weak potential modulation has been calculated for a 3DEG in Refs. 14 and 15 and for a 2DEG in Refs. 16-18. In Ref. 18 the energy spectrum with a Kronig-Penney $1 \mathrm{D}$ periodic potential was also considered.

The qualitative differences in the energy spectrum with and without a modulation are also reflected in the density of states (DOS), $D(E)=\left(1 / 2 \pi l^{2}\right) \sum_{n, k_{y}} \delta\left(E-E_{n, k_{y}}\right)$, which is expressed per unit surface. With $t=K x_{0}$ the DOS takes the form

$$
D(E) / D_{0}=\frac{\hbar \omega_{c}}{2 \pi} \sum_{n=0}^{\infty} \int_{0}^{\pi} d t \delta\left(E-E_{n, t}\right),
$$

with $D_{0}=m^{*} / \pi \hbar^{2}$ the DOS of a 2 DEG at $B=0$. Numerical results are given in Fig. 1 for the following parameters: $V_{0}=1 \mathrm{meV}, a=3500 \AA$, and $\hbar \omega_{c}=1 \mathrm{meV}$. Note that the $\delta$ function DOS for $V_{0}=0$ is broadened into a band $^{2,10,11,19}$ with van Hove singularities at the edge of the Landau levels. The latter reflects the $1 \mathrm{D}$ nature of the electron motion in the bands. This can be understood by calculating the electron velocity corresponding to an eigenstate $\left|n, k_{y}\right\rangle$,

$$
v_{y}=\frac{1}{\hbar} \frac{\partial E_{n k_{y}}}{\partial k_{y}}=-\frac{2 V_{0}}{\hbar K} u e^{-u / 2} L_{n}(u) \sin \left(K x_{0}\right),
$$

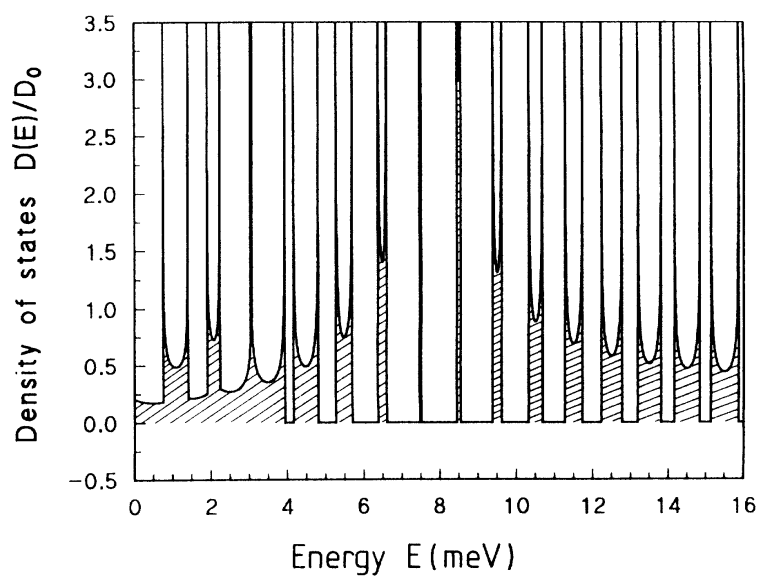

FIG. 1. The density of states (in units of zero magnetic-field value) of a $2 D E G$ modulated by a $1 D$ potential with strength $V_{0}=1 \mathrm{meV}$ and period $a=3500 \AA$. The magnetic field is such that $\hbar \omega_{c}=1 \mathrm{meV}$. 
while $v_{x}=0$. In the absence of the modulation $v_{x}=v_{y}=0$ for a Landau state, the DOS consists of $\delta$ functions, and the different Landau states can be interpreted as OD states localized in space where transport will occur through hopping between such states. Note that in Fig. 1, for $E<3 \mathrm{meV}$, the different Landau levels overlap and the results should be considered only as indicative. In practical expressions we need the DOS at the Fermi energy which is well beyond this regime, typically $E_{F} \sim 10$ meV.

The number of available states per Landau level $1 / 2 \pi l^{2}$ is the same for each Landau level independent of the index $n$. As a consequence, the average amplitude of the DOS of a Landau level will be small if it is considerably broadened. Therefore quantities which depend on the width of a Landau level will oscillate out of phase with quantities which depend on the magnitude of the DOS at a specific energy (i.e., the Fermi energy).

Recent magnetocapacitance measurements by Weiss et al..$^{20}$ reflect directly the oscillatory broadening of the DOS as due to a 1D modulation potential. The width of the Landau level $n$ is given by $2 V_{0} e^{-u / 2}\left|L_{n}(u)\right|$ and is plotted in Fig. 2 (solid curve) for $n=n_{F}$ where $n_{F}=E_{F} / \hbar \omega_{c}$ is the Landau level index at the Fermi energy. Because $n_{F}$ is taken as an integer, the plotted bandwidth exhibits a step each time a new Landau level moves through the Fermi level. For the system under consideration the magnetic field is small, and in order to make an estimate of the position of the minima and maxima of the bandwidth we will take the large $n$ limit of the Laguerre polynomial, i.e., $e^{-u / 2} L_{n}(u)$ $=\left(\pi^{2} n u\right)^{-1 / 4} \cos (2 \sqrt{n u}-\pi / 4)+O\left(1 / n^{3 / 4}\right)$, which is zero when $u=(1 / n)\left[\pi / 2\left(i+\frac{3}{4}\right)\right]^{2}, i=0,1,2, \ldots$, and maximum for $u=(1 / n)\left[\pi / 2\left(i+\frac{1}{4}\right)\right]^{2}$. Noting that the cyclotron radius $R_{c}=l \sqrt{2 n_{F}+1}$ with $n_{F}$ the Landau index at the Fermi level, we obtain $2 R_{c} / a=i+\phi$, where $\phi=\frac{1}{4}$, for maximum bandwidth and $\phi=\frac{3}{4}$ for flat band. For the physical parameters of Fig. 2 (i.e., $n_{e}=3 \times 10^{11}$ $\mathrm{cm}^{-2}$ and $a=3500 \AA$ ) we obtain for the position of the extrema $B(T)=0.516 /(i+\phi)$ which results into the magnetic-field values (1) for maximum band width $B(T)=2.06,0.413,0.229,0.159,0.121,0.0983, \ldots ;$ and

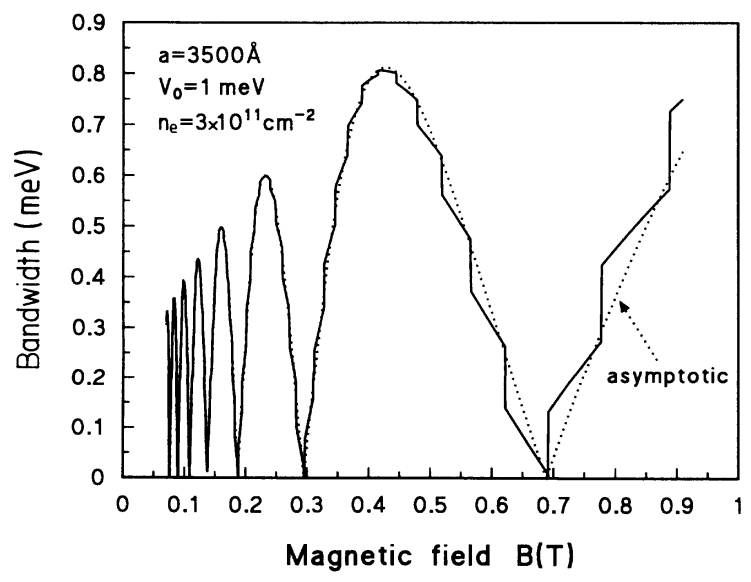

FIG. 2. The width of the Landau level at the Fermi energy as function of the magnetic field.
(2) for minimum bandwidth $B(T)=0.688,0.295,0.188$, $0.138,0.109,0.0897, \ldots$. These values agree very well with the position of the maxima and minima in the bandwidth shown in Fig. 2. Even for large magnetic fields the effect of the modulation potential on the Landau levels is nonzero, i.e., in the limit $a>>\sqrt{2 n} \pi l$ (or equivalently $\left.a \gg \pi R_{c}\right)$ the bandwidth is given by $2 V_{0} n_{F} !\left[1-\left(n_{F}+1 / 2\right) u+\cdots\right]$.

A more pictorial picture for the above resonance condition can be presented by giving a classical description in real space. The classical equation of motion of an electron in a magnetic field is $x(t)=x_{0}+R_{c} \sin \omega_{c} t$, with $R_{c}$ the radius and $x_{0}$ the orbit center. For our purpose the motion in the $y$ direction is not relevant. The increase of the average energy of this cyclotron motion due to the periodic potential is

$$
\Delta E\left(x_{0}\right)=\frac{1}{t_{0}} \int_{0}^{t_{0}} d t V_{0} \cos [K x(t)],
$$

where $t_{0}$ is the period of the motion. Inserting the expression for $x(t)$ into Eq. (5a) we obtain

$$
\Delta E\left(x_{0}\right)=V_{0} J_{0}\left(K R_{c}\right) \cos \left(K x_{0}\right),
$$

with $J_{0}(x)$ the Bessel function of order zero. Note that the energy does not depend on the position of the center of the cyclotron orbit when $J_{0}\left(K R_{c}\right)=0$. For $K R_{c}$ $\gg 1$ we obtain $\Delta E\left(x_{0}\right) \simeq V_{0}\left(2 / \pi K R_{c}\right)^{1 / 2} \cos \left(K R_{c}\right.$ $-\pi / 4) \cos \left(K x_{0}\right)$, which leads to the previous results for the condition of maximum bandwidth and flat band. This asymptotic expression is depicted in Fig. 2 by the dashed line and is surprisingly very close to the quantum-mechanical result.

In practical systems there will always be some broadening due to the presence of scattering centers. We assume a Lorentzian broadening of the $\delta$ function, i.e., $\delta(E) \rightarrow(1 / \pi) \Gamma /\left(E^{2}+\Gamma^{2}\right)$, for simplicity of zero shift and of constant width $\Gamma$ since we are considering the experimentally weak magnetic fields $(B<0.4 \mathrm{~T})$. Then Eq. (3) becomes

$$
D(E)=D_{0} \frac{\hbar \omega_{c}}{2 \pi} \sum_{n=0}^{\infty} \frac{1}{\pi} \int_{0}^{\pi} d t \frac{\Gamma}{\left(E-E_{n, t}\right)^{2}+\Gamma^{2}},
$$



FIG. 3. The same as Fig. 1 but now for a nonzero broadening of $\Gamma=1 \mathrm{~K}$ (solid curve) and $\Gamma=2 \mathrm{~K}$ (dashed curve). 
where $E_{n, t}$ is given by Eq. (2) with the substitution $t=2 \pi l^{2} k_{y} / a$. Because we are not interested in effects related to localization we take $D(E)=0$ for $E<0$. The effect of broadening on the DOS is shown in Fig. 3 where $D(E) / D_{0}$ is plotted as a function of the magnetic field for two values of the broadening $\Gamma=1$ and $2 \mathrm{~K}$ (note that 1 $\left.\mathrm{K} \approx 0.086 \mathrm{meV} / k_{B}\right)$. The qualitative behavior of the DOS is essentially the same if we assume a Gaussian broadening.

\section{THERMODYNAMICS}

Equilibrium thermodynamical properties provide information on the density of states, which does not directly involve any electron scattering mechanisms. The effect of scattering on the DOS will be assumed to be of secondary importance. The information on the DOS is indirect because any thermodynamic quantity involves an integral over the DOS.

Consider a 2DEG of noninteracting electrons, in the $(x, y)$ plane, which is modulated periodically (along the $x$ axis) by a weak 1D electrical potential. The conduction band is taken to be parabolic and spherically symmetric and a magnetic field is applied to the 2DEG along the $z$ axis. Spin degeneracy is included but not spin splitting, which is negligibly small for the considered $B$ fields. In the absence of any modulation potential a detailed study of the magnetization, specific heat, magnetothermal effect, and the thermoelectric power of a 2DEG in a strong magnetic field was given by Zawadzki and Lassnig. ${ }^{21}$ Here we will generalize this study to the case where a $1 \mathrm{D}$ modulation potential is present and we will be interested in not too large magnetic fields.

All thermodynamical properties of a system can be obtained as derivatives of the free energy. For a system of noninteracting Fermi particles the free energy per unit volume is given by ${ }^{21,22}$

$F=n_{e} \mu^{*}-2 k_{B} T \int_{0}^{\infty} d E D(E) \ln \left(1+e^{\left(\mu^{*}-E\right) / k_{B} T}\right)$,

where the chemical potential $\mu^{*}$ is determined by the electron density $n_{e}=2 \int_{0}^{\infty} d E f(E) D(E)$, with $f(E)$ $=1 /\left(e^{\beta\left(E-\mu^{*}\right)}+1\right)$ the Fermi-Dirac distribution function, $\beta=1 / k_{B} T$ the inverse temperature, and where the factor 2 accounts for spin degeneracy. Using Eq. (3) for $D(E)$ we have

$$
n_{e} \pi l^{2}=\sum_{n=0}^{\infty} \frac{1}{\pi} \int_{0}^{\pi} d t f\left(E_{n, t}\right)
$$

which has to be solved numerically in order to obtain the chemical potential $\mu^{*}$. The magnetic-field dependence of the correction to the chemical potential due to the weak 1D modulation: $\Delta E_{F}=\mu^{*}\left(V_{0}\right)-\mu^{*}\left(V_{0}=0\right)$ is shown in Fig. 4 for $a=3500 \AA, V_{0}=1 \mathrm{meV}$, and $n_{e}=3 \times 10^{11} \mathrm{~cm}^{-2}$ (for these values the chemical potential is about $\mu^{*} \approx 10.85 \mathrm{meV}$ in the magnetic-field range shown in Fig. 4). From Fig. 4 we see that (1) the 1D modulation induces weak oscillations in $\mu^{*}$ of order $0.1 \%$ for $B<0.3 \mathrm{~T}$ whose amplitude has a weak temperature dependence; (2) for $B>0.3 \mathrm{~T}$ the $T=2 \mathrm{~K}$ result shows $\mathrm{SdH}$ oscillations,

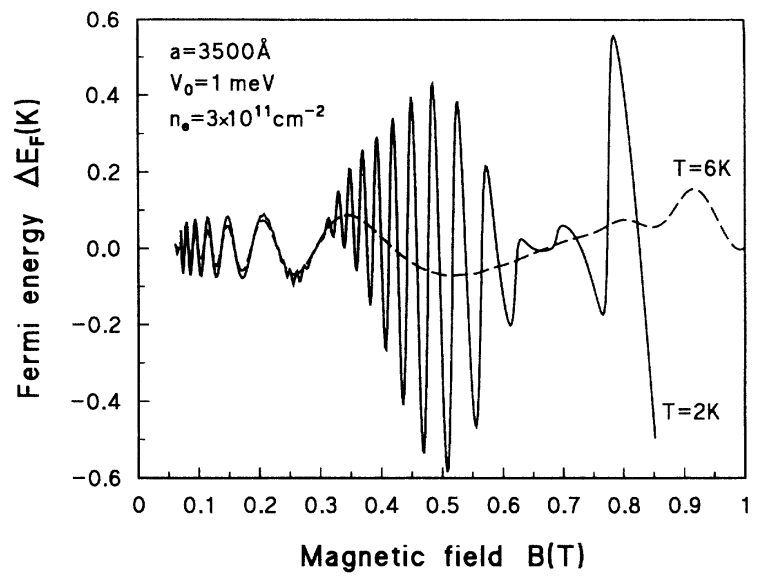

FIG. 4. The change of the Fermi energy due to the 1D modulation as function of the magnetic field for two values of the temperature.

which for $T=6 \mathrm{~K}$ are all damped out, and their amplitude is modulated by these oscillations; and (3) $\Delta E_{F}=0$ when the bandwidth is zero (see Fig. 2) as one expects. One period in bandwidth oscillation corresponds to one period in $\Delta E_{F}$ oscillation, and because $\Delta E_{F}$ oscillates around zero we have the remarkable result that also $\Delta E_{F}=0$ when the bandwidth is maximum.

From Eq. (7) we calculate the electronic contribution of the 2DEG to the magnetization $M=-\partial F / \partial B$, the susceptibility $\chi=-\partial^{2} F / \partial B^{2}$, and the specific heat $C=-T \partial^{2} F / \partial T^{2}$. Any changes in the DOS due to the 1D modulation will be reflected in changes in the different thermodynamical quantities. The results are shown in Fig. 5. We scaled all quantities per electron and in units of the effective Bohr magneton $\mu_{B}^{*}=e \hbar / 2 m^{*} c=0.87 \mathrm{meV} / \mathrm{T}$, which leads to the natural units $M_{0}=\chi_{0}=n_{e} \mu_{B}^{*}$ and which implies that $M / M_{0}$ is the magnetization per electron in units of the effective Bohr magneton and $\chi / \chi_{0}$ is the susceptibility per electron in units of the effective Bohr magneton per tesla. For our numerical calculation we took the parameters for GaAs with the effective mass $m^{*} / m_{e}=0.066$. The expression which was calculated numerically is the free energy per electron,

$F / n_{e}=\mu^{*}-\frac{k_{B} T}{n_{e} \pi l_{B}^{2}} \sum_{n=0}^{\infty} \frac{1}{\pi} \int_{0}^{\pi} d t \ln \left(1+e^{\left.\left(\mu^{*}-E_{n, t}\right) / k_{B} T\right)}\right) ;$

its numerical differentiation gave the different thermodynamic quantities.

A comparison between Fig. 4 and Figs. 5(a) and 5(b) shows that (1) $\Delta E_{F}$ and the magnetization oscillate in phase while the corresponding $\mathrm{SdH}$ oscillations for the $T=2 \mathrm{~K}$ curve are $180^{\circ}$ out of phase, and (2) $\Delta E_{F}$ and the susceptibility oscillations are shifted by $90^{\circ}$ as well as the corresponding $\mathrm{SdH}$ oscillations for $T=2 \mathrm{~K}$. The specific heat is measured from the result at zero modulation. Because the electron contribution to the specific heat increases with decreasing temperature we have plotted 
$\triangle C T$ per electron. The corresponding result is shown in Fig. 5(c), where the oscillations are in phase with those of the oscillations in the bandwidth.

\section{ELECTRICAL MAGNETOTRANSPORT}

\section{A. Basic expressions}

For weak electric fields $\mathbf{E}$, i.e., for linear responses, and weak scattering potentials the conductivity tensor
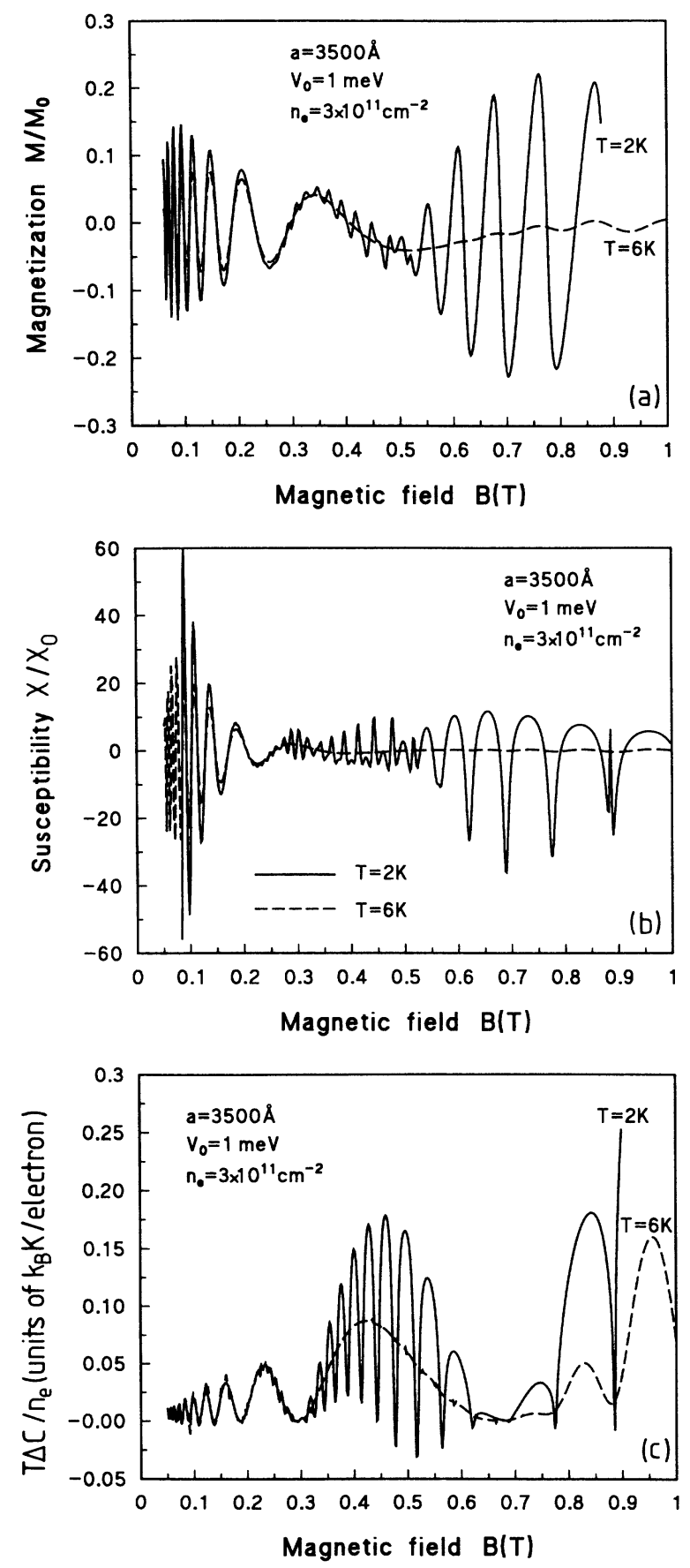

FIG. 5. The thermodynamic quantities (per electron): (a) the magnetization, (b) the susceptibility, and (c) the correction of the specific heat (multiplied with temperature) due to the 1D modulation as function of the magnetic field for $T=2 \mathrm{~K}$ (solid curves) and $T=6 \mathrm{~K}$ (dashed curves). $\sigma_{\mu \nu}(\omega)$, in the one-electron approximation, has been evaluated in Ref. 22; $\sigma_{\mu \nu}(\omega)=\sigma_{\mu \nu}^{d}(\omega)+\sigma_{\mu \nu}^{n d}(\omega), \mu, \nu=x, y, z$. $\sigma_{\mu \nu}^{d}(\omega)$ stems from the diagonal part $\rho^{d}$ of the density operator $\rho$. In any basis $\left\langle J_{\mu}^{d}\right\rangle=\operatorname{Tr}\left(\rho^{d} J_{\mu}\right)=\sigma_{\mu \nu}^{d} E_{\nu}$, where $J_{\mu}$ is the current density, and $\sigma_{\mu \nu}^{n d}(\omega)$ comes from the nondiagonal part of $\rho\left(\rho=\rho^{d}+\rho^{n d}\right)$. In general, $\sigma_{\mu \nu}^{d}(\omega)=\sigma_{\mu \nu}^{\mathrm{dif}}(\omega)+\sigma_{\mu \nu}^{\mathrm{col}}(\omega)$, where $\sigma_{\mu \nu}^{\mathrm{dif}}(\omega)$ indicates diffusion contributions and $\sigma_{\mu \nu}^{\text {col }}(\omega)$ collisional contributions. For dc conduction we have [cf. Eq. (2.55) of Ref. 23]

$$
\sigma_{\mu \nu}^{\mathrm{dif}}(0)=\frac{\beta e^{2}}{\Omega} \sum_{\zeta} f_{\zeta}\left(1-f_{\zeta}\right) \tau\left(E_{\zeta}\right) v_{\mu}^{\zeta} v_{v}^{\zeta},
$$

provided that the scattering is elastic or quasielastic, and [cf. Eq. (2.84) of Ref. 22]

$$
\sigma_{\mu \mu}^{\mathrm{col}}(0)=\frac{e^{2}}{2 \Omega} \sum_{\zeta \zeta^{\prime}} f_{\zeta^{\prime}}\left(1-f_{\zeta^{\prime}}\right) W_{\zeta \zeta^{\prime}}\left(\alpha_{\mu}^{\zeta}-\alpha_{\mu}^{\left.\zeta^{\prime}\right)^{2}},\right.
$$

both for elastic $\left(f_{\zeta}=f_{\zeta^{\prime}}\right)$ and inelastic $\left(f_{\zeta^{\prime}} \neq f_{\zeta^{\prime}}\right)$ scattering. $W_{\zeta \xi^{\prime}}$ is the transition rate between the one-electron states $\left|\zeta^{\prime}\right\rangle$ and $\left|\xi^{\prime}\right\rangle, \Omega$ the volume of the system, $e$ the electron charge, $\tau(E)$ the relaxation time, and $\alpha_{\mu}^{\zeta}=\left\langle\zeta\left|r_{\mu}\right| \zeta\right\rangle$ the mean value of the $\mu$ component of the position operator when the electron is in state $|\zeta\rangle$ and has velocity $v_{\mu}^{\zeta}=\left\langle\zeta\left|v_{\mu}\right| \zeta\right\rangle$.

Note that a diffusion contribution to the conductivity will be present when the electron states have a nonzero velocity. This contribution is also called band conduction and will be zero for localized states. For $|\zeta\rangle=|\mathbf{k}\rangle$, i.e., for free or nearly free electrons, Eq. (10) gives the standard semiclassical expression. This conductivity decreases with increasing impurity scattering. On the other hand, the collisional contribution describes the migration of the center of the cyclotron orbit due to scattering with impurities. Equation (11) is absent in a semiclassical treatment ${ }^{9}$ and describes transport through localized states (e.g., hopping). ${ }^{24,25}$ In the case of electrons in a magnetic field and in the absence of any modulation potential conduction occurs through hopping between cyclotron orbits which are centered at different positions in space. In this case impurity scattering helps the conduction and this contribution increases with increasing impurity scattering.

The nondiagonal contribution $\sigma_{\mu v}^{n d}(\omega)$ to the conductivity is given by Eq. (3.24) of Ref. 23 and reads

$$
\begin{aligned}
\sigma_{\mu v}^{n d}(\omega)=\frac{i \hbar e^{2}}{\Omega} \sum_{\zeta \neq \zeta^{\prime}} & f_{\zeta}\left(1-f_{\zeta^{\prime}}\right)\left\langle\zeta\left|v_{\mu}\right| \zeta^{\prime}\right\rangle\left\langle\zeta^{\prime}\left|v_{v}\right| \zeta\right\rangle \\
& \times \frac{1-e^{\beta\left(E_{\zeta}-E_{\zeta^{\prime}}\right)}}{E_{\zeta}-E_{\zeta^{\prime}}} \\
& \times \lim _{\epsilon \rightarrow 0} \frac{1}{E_{\zeta}-E_{\zeta^{\prime}}+\hbar \omega+i \epsilon} .
\end{aligned}
$$

If we use the identity $f_{\zeta}\left(1-f_{\xi^{\prime}}\right) \exp \left[\beta\left(E_{\xi^{\prime}}-E_{\xi^{\prime}}\right)\right]$ $=f_{\xi^{\prime}}\left(1-f_{\zeta}\right)$, Eq. (12) takes the form of the well-known Kubo-Greenwood formula.

A factor of 2 has to be included in Eqs. (10)-(12) if spin degeneracy is assumed. The above formulas have been successfully applied to various situations of elec- 
tronic transport: ${ }^{24,26}$ hopping conduction and magnetophonon resonances, quantum Hall effect, AharonovBohm effect, etc. . .

The resistivity tensor $\rho_{\mu v}$ is given in terms of the conductivity tensor $\rho=\sigma^{-1}$. We will use the standard expressions $\rho_{x x}=\sigma_{y y} / S, \rho_{y y}=\sigma_{x x} / S$, and $\rho_{y x}=-\rho_{x y}$ $=-\sigma_{y x} / S$, where $S=\sigma_{x x} \sigma_{y y}-\sigma_{x y} \sigma_{y x}$.

\section{B. The conductivity tensor}

First the diffusive contribution to the conductivity is evaluated. We are interested in weak modulation potentials, which implies that in Eq. (10) we may use $|\zeta\rangle=\left|n, k_{y}\right\rangle$ as the unperturbed 2D Landau states with the velocity given by Eq. (4). Since $v_{x}=0$ we have $\sigma_{x x}^{\mathrm{dif}}=0$, while

$$
\begin{aligned}
\sigma_{y y}^{\mathrm{dif}}= & 2 \frac{e^{2}}{h} \frac{2 \pi^{2}}{\hbar} \frac{l^{2}}{a^{2}} \tau V_{0}^{2} e^{-u} \\
& \times \sum_{n=0}^{\infty}\left[L_{n}(u)\right]^{2}\left(-\frac{\partial f(E)}{\partial E}\right)_{E=E_{n}}
\end{aligned}
$$

to order $V_{0}^{2}$. For convenience we took a constant scattering time $\tau=\tau(E)=\mu m^{*} c / e$ ( $\mu$ is the mobility at zero magnetic field) independent of energy and Landau-level index $n$, which for weak magnetic fields is a reasonable approximation. In our previous works, Refs. 10 and 13, a factor of 2 was missing in the final expression for the diffusion contribution to the conductivity. The corresponding result of Ref. 2, to order $V_{0}^{2}$, reduces to Eq. (13).

Next we calculate the collisional contribution to the conductivity. To be correct to order $V_{0}^{2}$ we have to include the correction to the unmodulated eigenstate $\left|n, k_{y}\right\rangle^{0}$ due to the weak 1D modulation; to first order in perturbation theory the corrected eigenstate is given by

$$
\left|n, k_{y}\right\rangle=\left|n, k_{y}\right\rangle^{0}+\sum_{n^{\prime} \neq n} \frac{V_{n, n^{\prime}}}{E_{n}-E_{n^{\prime}}}\left|n^{\prime}, k_{y}\right\rangle^{0},
$$

with the matrix elements

$$
\begin{aligned}
V_{n, n^{\prime}}= & V_{0}\left(\frac{n^{\prime} !}{n !}\right]^{1 / 2} e^{-u / 2_{u}\left(n-n^{\prime}\right) / 2} L_{n^{\prime}}^{n-n^{\prime}}(u) \\
& \times \operatorname{Re}\left(i^{n-n^{\prime}} e^{i K x_{0}}\right) .
\end{aligned}
$$

In the following we will need the matrix elements

$$
{ }^{0}\left\langle n, k_{y}\left|e^{i \mathbf{q} \cdot \mathbf{r}}\right| n^{\prime}, k_{y}^{\prime}\right\rangle^{0}=\left\{\begin{array}{l}
\delta_{k_{y}^{\prime}, k_{y}-q_{y}} e^{-i \Theta-\gamma / 2}\left[\frac{2^{n} n !}{2^{n^{\prime}} n^{\prime} !}\right]^{1 / 2} v^{n^{\prime}-n} L_{n}^{n^{\prime}-n}(\gamma), \quad n \leq n^{\prime} \\
\delta_{k_{y}^{\prime}, k_{y}-q_{y}} e^{-i \Theta-\gamma / 2}\left[\frac{2^{n} n^{\prime} !}{2^{n^{\prime}} n !}\right]^{1 / 2}\left(-v^{*}\right)^{n-n^{\prime}} L_{n^{\prime}}^{n-n^{\prime}}(\gamma), \quad n \geq n^{\prime}
\end{array}\right.
$$

with $\gamma=l^{2}\left(q_{x}^{2}+q_{y}^{2}\right) / 2=l^{2} q^{2} / 2, \quad \Theta=l^{2} q_{x}\left(-k_{y}+q_{y} / 2\right)$, $v=l\left(q_{y}+i q_{x}\right) / 2$, and $L_{n}^{n^{\prime}}(x)$ is the Laguerre polynomial. For completeness we mention that

$$
\begin{aligned}
& { }^{0}\left\langle n, k_{y}|x| n, k_{y}\right\rangle^{0}=x_{0}, \\
& { }^{0}\left\langle n, k_{y}\left|v_{x}\right| n^{\prime}, k_{y}\right\rangle^{0} \\
& \quad=i\left(\frac{\hbar \omega_{c}}{2 m^{*}}\right)^{1 / 2}\left(-\sqrt{n+1} \delta_{n^{\prime}, n+1}+\sqrt{n} \delta_{n^{\prime}, n-1}\right),
\end{aligned}
$$

$$
\begin{aligned}
& { }^{0}\left\langle n, k_{y}\left|v_{y}\right| n, k_{y}\right\rangle^{0} \\
& =\left(\frac{\hbar \omega_{2}}{2 m^{*}}\right)^{1 / 2}\left(\sqrt{n+1} \delta_{n^{\prime}, n+1}+\sqrt{n} \delta_{n^{\prime}, n-1}\right) .
\end{aligned}
$$

We assume that electrons are scattered elastically by randomly distributed impurities. This type of scattering is dominant at low temperatures. For the evaluation of the relevant matrix elements, which enter the conductivity expressions (10)-(12), we use the electron wave functions (14) in the absence of any impurity scattering. We take the screened impurity potential $U(\mathbf{r}-\mathbf{R})=\left(e^{2} /\right.$ $\epsilon|\mathbf{r}-\mathbf{R}|) \exp \left(-k_{s}|\mathbf{r}-\mathbf{R}|\right)$ and we write it in terms of its Fourier transform $U_{q}=\left(2 \pi e^{2} / \epsilon\right) \sqrt{q^{2}+k_{s}^{2}}$, where $\mathbf{r}$ and $\mathbf{R}$ are the position of the electron and of the impurity, respectively, $k_{s}$ is the screening wave vector, and $\epsilon$ is the dielectric constant of the material the electron is moving in. by

The scattering rate $W_{\zeta \zeta^{\prime}}$, appearing in Eq. (11), is given

$$
\begin{gathered}
W_{\zeta \zeta^{\prime}}=\sum_{\mathrm{q}}\left|U_{q}\right|^{2}\left|\left\langle n, k_{y}\left|e^{i \mathbf{q} \cdot(\mathbf{r}-\mathbf{R})}\right| n^{\prime}, k_{y}^{\prime}\right\rangle\right|^{2} \\
\quad \times \delta\left(E_{n, k_{y}}-E_{n^{\prime}, k_{y}^{\prime}}\right) .
\end{gathered}
$$

Now we perform an average over a random distribution of impurities and denote $N_{I}$ as the 2D impurity density. As an example we calculate the contribution of the unperturbed part of the wave function $\left|n, k_{y}\right\rangle^{0}$ to the scattering rate

$$
\begin{aligned}
& W_{\zeta \zeta^{\prime}}^{(0)}= W_{n k_{y}, n^{\prime} k_{y}^{\prime}}^{(0)} \\
&=\frac{2 \pi N_{I}}{A_{0} \hbar} \sum_{\mathbf{q}}\left|U_{q}\right|^{2}\left|J_{n n^{\prime}}(\gamma)\right|^{2} \\
& \qquad \quad \times \delta\left(E_{n k_{y}}-E_{n^{\prime} k_{y}^{\prime}}\right) \delta_{q_{y}, k_{y}-k_{y}^{\prime}},
\end{aligned}
$$

with

$\left|J_{n n^{\prime}}(\gamma)\right|^{2}=\frac{n !}{n^{\prime} !} e^{-\gamma} \gamma^{n-n^{\prime}}\left[L_{n^{\prime}}^{n-n^{\prime}}(\gamma)\right]^{2}, \quad n^{\prime} \leq n$

where $A_{0}=L_{x} L_{y}$ is the surface area of the 2D system.

Inserting Eq. (19) into Eq. (11) we obtain (with $\left.\Omega \rightarrow A_{0}\right)$ for elastic scattering $\left(f_{\zeta}=f_{\zeta^{\prime}}\right)$ 


$$
\sigma_{x x}^{\mathrm{col}, 0}=\frac{2 \pi}{\hbar} \beta e^{2} l^{4} \frac{N_{I}}{A_{0}} \sum_{n, k_{y}} \sum_{n^{\prime}, k_{y}^{\prime}} \sum_{\mathrm{q}}\left|U_{q}\right|^{2}\left|J_{n n^{\prime}}(\gamma)\right|^{2} q_{y}^{2} f_{n k_{y}}\left(1-f_{n k_{y}}\right) \delta\left(E_{n k_{y}}-E_{n^{\prime}, k_{y}^{\prime}}\right) \delta_{q_{y}, k_{y}-k_{y}^{\prime}},
$$

with $f_{n, k_{y}}=f\left(E_{n, k_{y}}\right)$ the Fermi-Dirac distribution function. This expression can be cast into the familiar form of DoS squared by noting that $\delta\left(E_{n k_{y}}-E_{n^{\prime} k_{y}^{\prime}}\right)=\int d E \delta\left(E-E_{n k_{y}}\right) \delta\left(E-E_{n^{\prime} k_{y}^{\prime}}\right)$ and consequently Eq. (21) contains a product of two density of states, $D(E) \sim \sum_{n, k_{y}} \delta\left(E-E_{n k_{y}}\right)$. The factor $\exp (-\gamma)$, in Eq. (20), favors small values of $q_{x}$ and $q_{y}$. Expanding the $\delta$ function in powers of $q_{y}$, we find that all terms proportional to $q_{y}^{2 m+1}$ vanish identically and that the first significant term $\left(q_{y}^{2}\right)$ is about an order of magnitude smaller than the term with $q_{y}=0$. With $\Sigma_{\mathrm{q}} \rightarrow\left(A_{0} / 4 \pi l^{2}\right) \int d \phi d u$, where $q_{y}=q_{1} \sin \phi$, and the small- $q$ limit for $U_{q}^{2}$, i.e., $U_{q}^{2} \approx U_{0}^{2}=\left(2 \pi e^{2} / \epsilon k_{s}\right)^{2}$, which is valid for $q<<k_{s}$, we obtain

$$
\sigma_{x x}^{\mathrm{col}, 0} \approx \frac{e^{2}}{\hbar} \frac{N_{I} U_{0}^{2}}{A_{0}} \sum_{n, n^{\prime}, k_{y}}\left(n+n^{\prime}+1\right)\left[-\frac{\partial f(E)}{\partial E}\right]_{E=E_{n, k_{y}}} \delta\left(\left(n-n^{\prime}\right) \hbar \omega_{c}+\Delta_{k_{y}}\left[L_{n}(u)-L_{n^{\prime}}(u)\right]\right)
$$

where $\Delta_{k_{y}}=V_{0} \exp (-u / 2) \cos \left(K x_{0}\right)$. We now replace the $\delta$ function by a Lorentzian of zero shift and of constant width $\Gamma_{n}$ and from the sum over $n^{\prime}$ we keep only the dominant term $n^{\prime}=n$. Then with $\Sigma_{k} \rightarrow \int d k$, $L_{x}=N a$, and $0<x_{0}<L_{x}$ we get

$$
\sigma_{x x}^{\mathrm{col}, 0} \approx \frac{e^{2}}{h} \frac{N_{I} U_{0}^{2}}{\pi a} \sum_{n=0}^{\infty}(2 n+1) \frac{1}{\Gamma_{n}} I_{n}
$$

with

$$
I_{n}=\int_{0}^{a / l^{2}} d k_{y}\left(\frac{-\partial f(E)}{\partial E}\right) E=E_{n, k_{y}}
$$

and this is the result reported in Ref. 10. In the absence of any modulation and in the limit $T=0$, Eq. (23) becomes $\sigma_{x x}=\left(e^{2} / \hbar \pi^{2}\right)\left(n_{F}+1 / 2\right)$ when the Fermi energy is in the middle of Landau level $n_{F}$. This result was originally derived by Ando, Fowler, and Stern. ${ }^{27}$ In the following we will take $\Gamma_{n}=\Gamma$ independent of the Landau quantum number.

The perturbed wave function (14) will give an additional contribution to the scattering rate of order $V_{0}^{2}$, which must be included. This additional contribution was neglected in our earlier work ${ }^{10}$ and leads to the dominant $V_{0}^{2}$ contribution to $\sigma_{x x}^{\text {col }}$. Noting that the Landau levels at the Fermi energy are well separated, we include only the contribution from the nearest-neighbor Landau levels. A similar calculation as above leads to the final result

$$
\sigma_{x x}^{\mathrm{col}} \approx \frac{e^{2}}{h} \frac{N_{I} U_{0}^{2}}{\pi \Gamma a} \sum_{n=0}^{\infty}\left[(2 n+1) I_{n}+\Delta_{n}\right],
$$

with

$$
\begin{array}{r}
\Delta_{n}=2\left(\frac{V_{0}}{\hbar \omega_{c}}\right)^{2} u e^{-u}\{[ \\
\left.L_{n-1}^{1}(u)\right]^{2}+\left[L_{n}^{1}(u)\right]^{2} \\
\left.+L_{n-1}^{1}(u) L_{n}^{1}(u)\right\} J_{n},
\end{array}
$$

which is a reduced form of Eq. (6) of Ref. 13. In Eq. (26) we introduced

$$
J_{n}=\int_{0}^{a / l^{2}} d k_{y} \sin ^{2}\left(K x_{0}\right)\left[\frac{-\partial f(E)}{\partial E}\right]_{E=E_{n k_{y}}} .
$$

Next we evaluate the Hall conductivity (12) for zero frequency. Inserting Eqs. (17b) and (17c) into Eq. (12) and using the identity $f_{\zeta}\left(1-f_{\xi^{\prime}}\right) \exp \left[\beta\left(E_{\zeta}-E_{\xi^{\prime}}\right)\right]$ $=f_{\zeta^{\prime}}\left(1-f_{\zeta}\right)$, we obtain

$\sigma_{y x}=\frac{e^{2}}{h} \frac{2 l^{2}}{a} \sum_{n=0}^{\infty}(n+1) \int_{0}^{a / l^{2}} d k_{y} \frac{f_{n, k_{y}}-f_{n+1, k_{y}}}{\left[1+\lambda_{n} \cos \left(K x_{0}\right)\right]^{2}}$,

where $\lambda_{n}=\left(V_{0} / \hbar \omega_{c}\right) \exp (-u / 2) L_{n+1}^{-1}(u)$. In the limit $T \rightarrow 0$ and for $E_{n}<E_{F}<E_{n+1}$, Eq. (28) gives approximately $\sigma_{y x} \approx\left(e^{2} / h\right)(n+1)\left(1+3 \lambda_{n}^{2} / 2\right)$ when the denominator is expanded in powers of $\lambda_{n}$.

\section{Numerical results}

The above expressions for the conductivity, Eqs. (13), (25), and (28), are evaluated numerically and shown in Fig. 6(a) for two temperatures $T=2$ and $6 \mathrm{~K}$ for a $2 \mathrm{DEG}$ with electron density $n_{e}=3.10^{11} \mathrm{~cm}^{-2}$, mobility $\mu=10^{6}$ $\mathrm{cm}^{2} / \mathrm{V} \mathrm{s}$, 1D modulation strength $V_{0}=0.5 \mathrm{meV}$, and period $a=3500 \AA$. Note that $\sigma_{x x}=\sigma_{x x}^{\text {col }}$ and $\sigma_{y y}=\sigma_{y y}^{\text {col }}+\sigma_{y y}^{\text {diff. }}$. The correction to the conductivity due to the modulation potential $\Delta \sigma_{\mu \nu}=\sigma_{\mu \nu}\left(V_{0}\right)-\sigma_{\mu \nu}\left(V_{0}=0\right)$ is shown in Fig. 6(b). The different plots of $\Delta \sigma_{\mu v}$ are offset and the zero is indicated by the position of the horizontal arrows.

Note that the 1D modulation (1) gives a positive contribution to $\Delta \sigma_{x x}$ and $\Delta \sigma_{y y}$, while $\Delta \sigma_{y x}$ oscillates around zero; (2) $\Delta \sigma_{y y} \gg \Delta \sigma_{x x}$, which is a consequence of the fact that $\sigma_{x x}$ has only collisional contributions, while $\sigma_{y y}$, in addition to the collisional part, has contributions due to band conduction which are much larger; and (3) the oscillations in $\Delta \sigma_{y y}$ and $\Delta \sigma_{x x}$ are $180^{\circ}$ out of phase, while $\Delta \sigma_{y x}$ oscillates in phase with $\Delta \sigma_{x x}$.

The components of the resistivity tensor are shown in Fig. 7 (a) for $T=2$ and $6 \mathrm{~K}$. The contribution to $\rho_{\mu \nu}$ due to the modulation is shown in Fig. 7(b). Note that (1) the Weiss oscillations in $\rho_{x x}$ and $\rho_{y y}$ are out of phase; (2) the amplitude of the oscillations in $\Delta \rho_{x x}$ are considerably larger than those in $\Delta \rho_{y y}$ and $\Delta \rho_{x y} ;(3)$ the $T=2 \mathrm{~K}$ results exhibit SdH oscillations on top of the Weiss oscillations for $B>0.3 T$; (4) $\Delta \rho_{x y}$ oscillates in phase with $\Delta \rho_{x x}$, and (5) for small magnetic fields the minima in $\Delta \rho_{x x}$ are 
nonzero and thus the modulation gives an overall positive contribution to the resistivity which is absent in, e.g., the semiclassical treatment of Ref. 9. This effect is similar to the positive magnetoresistivity discussed by Středa and MacDonald, ${ }^{12}$ who attributed this to magnetic breakdown. This effect was also absent in our earlier treatment ${ }^{10}$ where only the contribution $\sigma_{x x}^{\text {col,0 }}$ was taken into account in our calculation.

The present theory also predicts Weiss oscillations in the Hall resistance as is shown in Fig. 7(b). They are pronounced when we plot the slope of the Hall resistance as function of the magnetic field which is shown in Fig. 8. At present no experimental results on $d R_{H} / d B$ are available. Such oscillations in $R_{H}$ are not in contradiction with the fact that the small magnetic-field Hall resistance is independent of scattering. The periodic modulation should not be viewed as an additional scattering mechanism, but rather as a means of modifying the energy spectrum which in turn will have an influence on the value of $R_{H}$. A similar situation occurs in heterostructures, where it was shown in Ref. 28 that doping with additional $\mathrm{Be}$ or $\mathrm{Si}$ impurities near the $2 \mathrm{DEG}$ is able to shift the quantum Hall plateaus. The underlying reason for such a
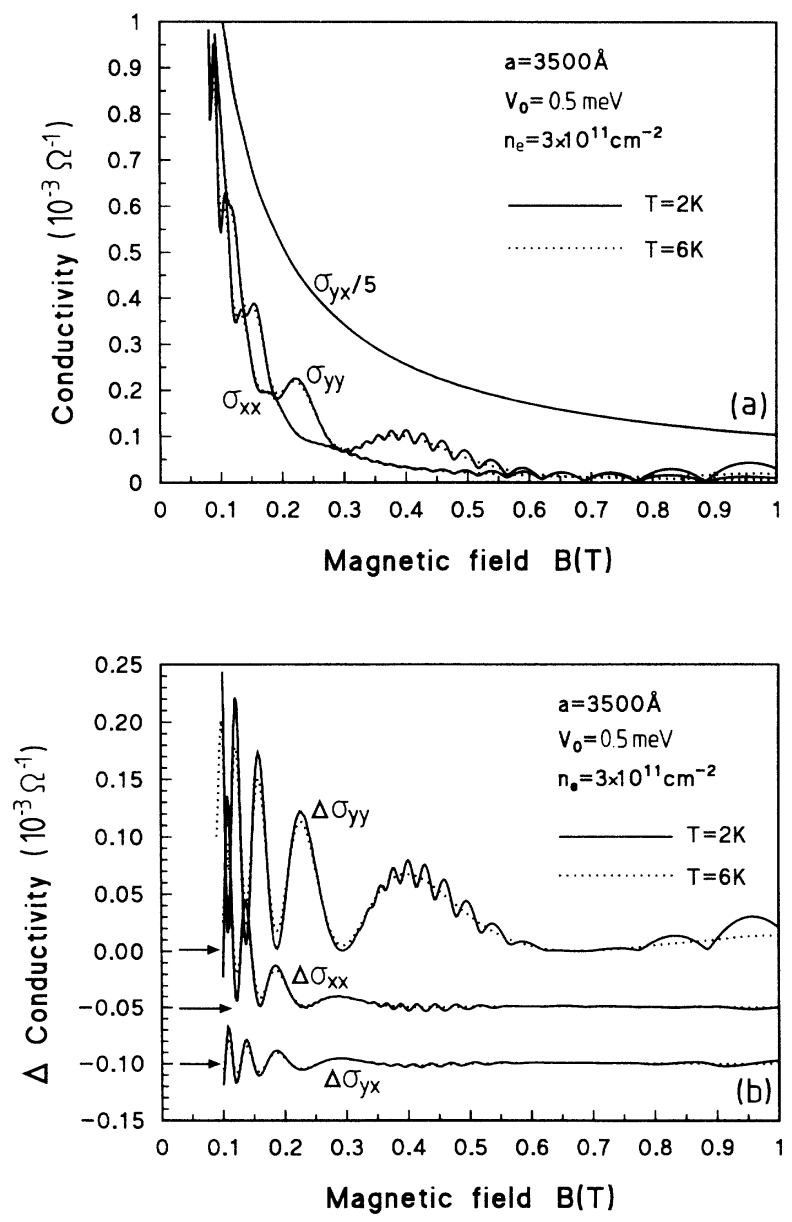

FIG. 6. The components of the conductivity tensor (a) as function of the magnetic field for $T=2 \mathrm{~K}$ (solid curves) and $T=6 \mathrm{~K}$ (dotted curves). The contribution due to the modulation is shown in (b), where the different curves are shifted vertically and the zero position is indicated by the arrows.
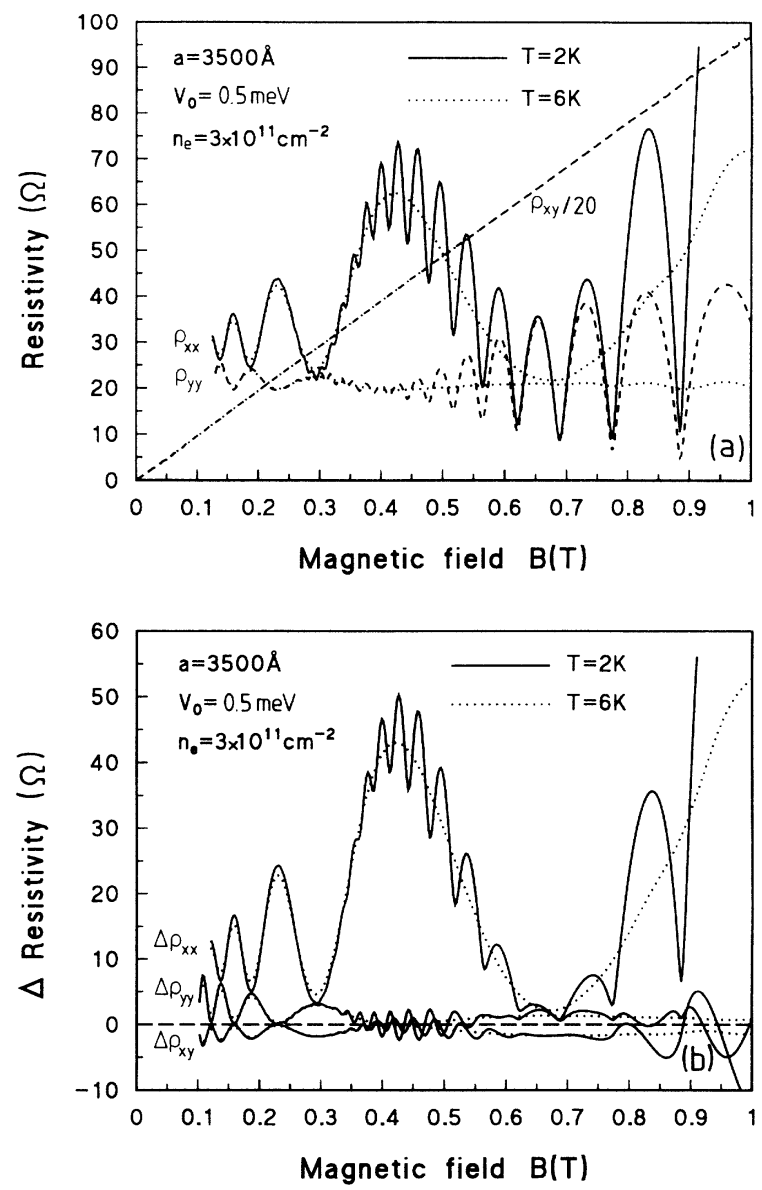

FIG. 7. The same as Fig. 6 but now for the resistivity tensor.

shift was the modification of the shape of the DOS of the Landau levels.

It should be noted that the effect of the 1D modulation potential on the electrical transport does not disappear with increasing magnetic field. To illustrate this we have shown in Fig. 9 the components of the conductivity tensor in the magnetic-field range $B: 1-3 \mathrm{~T}$. There is a

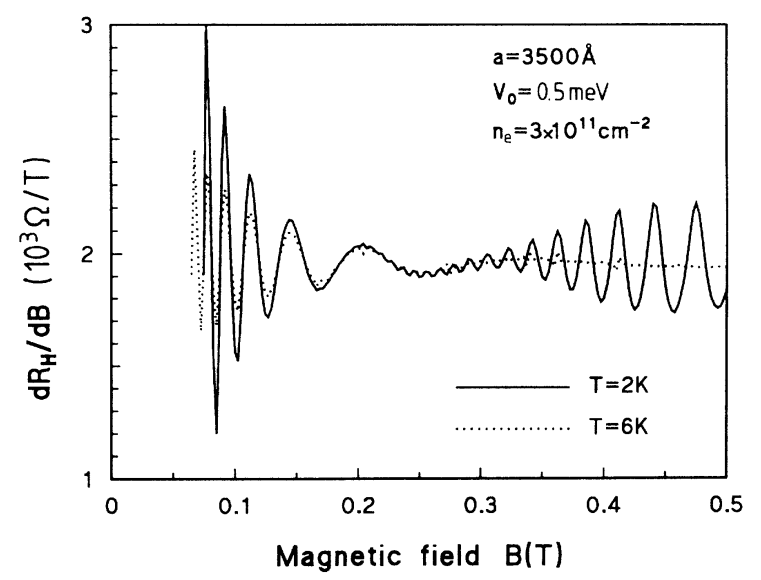

FIG. 8. The derivative of the Hall resistance with respect to the magnetic field for $T=2 \mathrm{~K}$ (solid curve) and $T=6 \mathrm{~K}$ (dashed curve). 


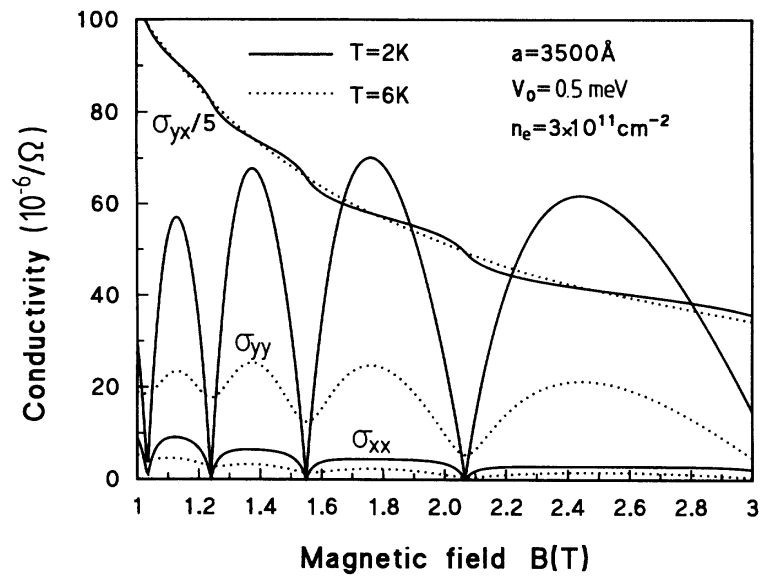

FIG. 9. The high magnetic-field behavior of the different components of the conductivity tensor for $T=2 \mathrm{~K}$ (solid curves) and $T=6 \mathrm{~K}$ (dotted curves).

strong asymmetry in the conductivity tensor where $\sigma_{y y} \gg \sigma_{x x}$. The reason is that the contribution to $\sigma_{y y}$ due to band conduction is much larger than the collisional one which decreases with increasing magnetic-field strength.

\section{Asymptotic expressions}

In this subsection we will look for simple analytic expressions for the results of the magnetoresistivity tensor. In view of the very good agreement, shown in Fig. 2, between the asymptotic result for the bandwidth and the quantum-mechanical result, we will be able to present asymptotic expressions for Eqs. (13) and (25) which approach our numerical results of Sec. IV C very closely. Furthermore, some of the existing results in the literature will be obtained as limiting cases.

In the asymptotic limit of many filled Landau levels (i.e., $n \gg 1$ ) we may replace $e^{-u / 2} L_{n}(u)$ by $\left(\pi^{2} n u\right)^{-1 / 4} \cos (2 \sqrt{n u}-\pi / 4)$ and take the continuum limit $\sum_{n=0}^{\infty} \rightarrow \int_{0}^{\infty} d E / \hbar \omega_{c}$ with the substitution $E=\hbar \omega_{c}\left(n+\frac{1}{2}\right), n u=\pi^{2}\left(R_{c} / a\right)^{2}$, and $E_{F} / R_{c}^{2}=\frac{1}{2} m \omega_{c}^{2}$.

First we consider the diffusion contribution Eq. (13) and obtain after the above substitutions

$$
\begin{aligned}
\sigma_{y y}^{\mathrm{dif}}= & \frac{e^{2}}{h} \frac{\pi}{\hbar} \frac{l^{2}}{a^{2}} \tau V_{0}^{2} \frac{\beta}{\sqrt{u \hbar \omega_{c}}} \\
& \times \int_{0}^{\infty} \frac{d E}{\sqrt{E}} \frac{\cos ^{2}\left(2 \sqrt{u / \hbar \omega_{c}} \sqrt{E}-\pi / 4\right)}{\cosh ^{2} \frac{\beta}{2}\left(E-E_{F}\right)},
\end{aligned}
$$

which under the assumption that $E_{F} \gg k_{B} T$ can be performed analytically and results in

$$
\frac{\sigma_{y y}^{\mathrm{dif}}}{\sigma_{0}}=\frac{V_{0}}{E_{F}} \frac{V_{0}}{\hbar \omega_{c}} \frac{2}{a k_{F}} F,
$$

with

$F=\frac{1}{2}\left[1-A\left(T / T_{a}\right)\right]+A\left(T / T_{a}\right) \cos ^{2}\left(2 \pi \frac{R_{c}}{a}-\pi / 4\right)$, where we defined the amplitude

$$
A(x)=\frac{x}{\sinh (x)},
$$

the characteristic temperature

$$
k_{B} T_{a}=\frac{\hbar \omega_{c}}{4 \pi^{2}} a k_{F}
$$

and $k_{F}=\sqrt{2 \pi n_{e}}=\sqrt{2 m^{*} E_{F}} / \hbar$ the Fermi wave vector. In the $T / T_{a} \rightarrow 0$ temperature limit the above expression, Eq. (30), was first obtained by Winkler, Kotthaus, and Ploog. ${ }^{3}$ The generalization to nonzero temperature was given by Davison et al ${ }^{29}$ who obtained Eq. (31) except for the nonoscillating term. In Ref. 29 it was found that the amplitude of the Weiss oscillations in the magnetoresistivity $\rho_{x x} / \rho_{0}=\left(\omega_{c} \tau\right)^{2} \sigma_{y y} / \sigma_{0}$ was accurately described by Eq. (30) where $\sigma_{0}=n_{e} e \tau / \mathrm{m}$.

The above expression is valid for $k_{B} T \gg \hbar \omega_{c} / 2 \pi^{2}$ and is not able to account for the $\mathrm{SdH}$ oscillations which are apparent in the numerical results for $T=2 \mathrm{~K}$ as depicted in Figs. 6 and 7. Therefore we use the well-known DOS (Ref. 27) for small magnetic fields,

$$
D(E)=\frac{m}{2 \pi \hbar^{2}}\left[1-2 e^{-\pi / \omega_{c} \tau_{f}} \cos \left(2 \pi E / \hbar \omega_{c}\right)+\cdots\right],
$$

with $\tau_{f}$ the quantum lifetime, also called single-particle relaxation time, which, depending on the type of scatterers, can be different ${ }^{30}$ from the scattering time obtained from the mobility. Using similar approximations as above and inserting the continuum approximation $\sum_{n=0}^{\infty} \rightarrow 2 \pi l^{2} \int_{0}^{\infty} d E D(E)$, we obtain the result

$$
\begin{aligned}
& \frac{\sigma_{y y}^{\mathrm{dif}}}{\sigma_{0}}=\frac{V_{0}}{E_{F}} \frac{V_{0}}{\hbar \omega_{c}} \frac{2}{a k_{F}}[F- 2 e^{-\pi / \omega_{c} \tau_{f}} A\left(T / T_{c}\right) \\
& \times \cos \left(\frac{2 \pi E_{F}}{\hbar \omega_{c}}\right) \\
&\left.\times \cos ^{2}\left(2 \pi \frac{R_{c}}{a}-\pi / 4\right)\right],
\end{aligned}
$$

where the amplitude of the SdH oscillations are determined by the well-known characteristic temperature

$$
k_{B} T_{c}=\frac{\hbar \omega_{c}}{2 \pi^{2}} .
$$

In the usual experimental situations we have $T_{a} / T_{c}=\frac{1}{2} a k_{F} \gg 1$ (e.g., for $n_{e}=3 \times 10^{11} \mathrm{~cm}^{-2}$ and $\left.a=3500 \AA, a k_{F} / 2=24\right)$ and as a consequence the $\mathrm{SdH}$ oscillations disappear much faster with temperature than the Weiss oscillations. For small magnetic fields, but such that $\omega_{c} \tau \gg 1$, the main contribution of the 1D modulation to the magnetoresistivity is given by $\Delta \rho_{x x}^{\mathrm{dif}} / \rho_{0}=\omega_{c}^{2} \tau^{2} \sigma_{y y}^{\mathrm{dif}} / \sigma_{0}$.

The physical reason for the difference in temperature scale between the amplitude of the Weiss oscillations and 
the SdH oscillations can be understood as follows. ${ }^{29}$ At nonzero temperature, electrons with an energy $k_{B} T$ around the Fermi energy $E_{F}$ contribute to conduction. $\mathrm{SdH}$ oscillations are a consequence of the discreteness of the Landau levels and thus they will be observable as long as the thermal broadening is smaller than the distance between two Landau levels, i.e., $k_{B} T \ll \hbar \omega_{c}$. The Weiss oscillations are determined by the size of the cyclotron orbit versus the period of the potential. The thermal smearing of the Fermi level $\delta E_{F}=k_{B} T$ will result in a smearing of the Fermi wave vector $\delta k_{F}=m k_{B} T / \hbar^{2} k_{F}$, and consequently the relevant cyclotron radius $R_{c}=\hbar k_{F} / e B$ will be smeared over the range $\delta R_{c}=k_{B} T / \hbar \omega_{c} k_{F}$. The Weiss oscillations will be observed as long as the spreading in the diameter of the contributing cyclotron orbits to the conduction is smaller than the period of the potential, i.e., $2 \delta R_{c} \ll a$, which results in the following condition on the temperature: $k_{B} T \ll \hbar \omega_{c} k_{F} a / 2$. This explains the factor $k_{F} a / 2$ difference in the temperature scale between the $\mathrm{SdH}$ and the Weiss oscillations.

For the collisional contribution to the magnetoconductivity, Eq. (25), we are able to obtain similar asymptotic expressions. The main contribution to the Weiss oscillations in $\sigma_{x x}^{\text {col }}$, are from the $\Delta_{n}$ term in Eq. (26). Therefore we take $V_{0} \rightarrow 0$ in Eq. (25) and apply the continuum limit to obtain

$$
\begin{aligned}
\sum_{n=0}^{\infty}(2 n+1) I_{n} & =\frac{a}{l^{2}} \frac{2}{\left(\hbar \omega_{c}\right)^{2}} \frac{1}{\beta} \ln \left(1+e^{\beta E_{F}}\right) \\
& \simeq \frac{a}{l^{2}} \frac{2 E_{F}}{\left(\hbar \omega_{c}\right)^{2}}, \quad E_{F} \gg k_{B} T,
\end{aligned}
$$

which leads to the standard expression $\sigma_{x x}^{(0)} / \sigma=1 /\left(\omega_{c} \tau\right)^{2}$ in the limit $\omega_{c} \tau \gg 1$ for zero modulation.
Using the corresponding asymptotic expression $e^{-u / 2 \sqrt{u}} L_{n}^{1}(u) \simeq 1 / \sqrt{\pi}(n / u)^{1 / 4} \sin (2 \sqrt{n u}-\pi / 4) \quad$ for the term $\Delta_{n}$, given by Eq. (26), and proceeding in the same way as for the diffusion contribution, we obtain

$$
\sigma_{x x}=\sigma_{x x}^{c o l}=\sigma_{x x}^{(0)}\left(1+\frac{V_{0}}{E_{F}} \frac{V_{0}}{\hbar \omega_{c}} \frac{3 a k_{F}}{4 \pi^{2}} C\right),
$$

where

$$
C=\frac{1}{2}\left[1-A\left(T / T_{a}\right)\right]+A\left(T / T_{a}\right) \sin ^{2}\left(2 \pi R_{c} / a-\pi / 4\right),
$$

which is of a similar form as Eq. (31) but the oscillatory term is now a $\sin ^{2} \theta$ instead of a $\cos ^{2} \theta$.

For the $y y$ component of the conductivity we have an additional contribution due to diffusive conduction,

$$
\sigma_{y y}=\sigma_{x x}+\sigma_{y y}^{\mathrm{dif}},
$$

and consequently $\sigma_{y y} \geq \sigma_{x x}$, which implies $\rho_{x x} \geq \rho_{y y}$. Note that $\rho_{y y}=\rho_{x x}$ when $\sigma_{y y}^{\mathrm{dif}}=0$, which occurs for $2 R_{c} / a=i+3 / 4$. The contribution due to the modulation to the collisional conductivity is related to the diffusion one by the factor

$$
\delta=\frac{\sigma_{x x}^{\mathrm{col}} / \sigma_{x x}^{(0)}-1}{\sigma_{y y}^{\mathrm{dif}} / \sigma_{x x}^{(0)}}=\frac{1}{\omega_{c}^{2} \tau^{2}} \frac{3 a^{2} k_{F}^{2}}{4 \pi^{2}} \frac{C}{F},
$$

which for $\mu=10^{6} \mathrm{~cm}^{2} / \mathrm{V} \mathrm{s}, a=3500 \AA$, and $n_{e}=3 \times 10^{11}$ $\mathrm{cm}^{-2}$ becomes $\delta=1.7,0.75$, and 0.20 for $B=0.1,0.2$, and $0.3 \mathrm{~T}$, respectively.

In Eq. (37) we took the limit $k_{B} T \gg \hbar \omega_{c}$, and as a consequence all $\mathrm{SdH}$ oscillations are completely damped out. Introducing Eq. (33) as the DOS and using the same asymptotic expressions for $L_{n}^{1}(u)$ and the continuum limit for the discrete sum over the Landau levels results in

$$
\begin{aligned}
\frac{\sigma_{x x}}{\sigma_{0}}=\frac{1}{\left(\omega_{c} \tau\right)^{2}}\{ & 1-2 e^{-\pi / \omega_{c} \tau_{f}} A\left(T / T_{c}\right) \cos \left[2 \pi \frac{E_{F}}{\hbar \omega_{c}}\right) \\
& \left.+\frac{V_{0}}{\hbar \omega_{c}} \frac{V_{0}}{E_{F}} \frac{3 a k_{F}}{4 \pi^{2}}\left[C-2 e^{-\pi / \omega_{c} \tau_{f}} A\left(T / T_{c}\right) \cos \left(2 \pi \frac{E_{F}}{\hbar \omega_{c}}\right) \sin ^{2}\left(2 \pi \frac{R_{c}}{a}-\pi / 4\right)\right]\right\} .
\end{aligned}
$$

At extremely small magnetic fields such that $\omega_{c} \tau<1$ the above approach is no longer valid because (1) intraLandau-level scattering becomes important; and (2) the distance between the Landau levels will be so small that $\hbar \omega_{c}<V_{0}$, and we are no longer allowed to apply perturbation theory for the modulation potential. An approach based on a Drude model as given by Středa and MacDonald $^{12}$ would be more appropriate in this case.

\section{THERMAL MAGNETOTRANSPORT}

In this section we calculate the thermal conductivity $\left(\kappa_{\mu \nu}\right)$ and thermopower $\left(S_{\mu \nu}\right)$ of the 2DEG modulated by a $1 D$ potential at very low temperatures when the impurity contribution to these coefficients is more important than the corresponding phonon contribution. The gen- eral expressions for these quantities were derived in Ref. 31 from linear-response theory in combination with the phenomenological transport equations for the electrical and thermal (energy) currents. For the 2DEG these expressions can be written in tensor form as follows:

$$
\begin{aligned}
& S_{\mu \nu}=\frac{1}{e T}\left(L^{(0)-1} L^{(1)}\right)_{\mu \nu}, \\
& \kappa_{\mu \nu}=\frac{1}{e^{2} T}\left[L_{\mu \nu}^{(2)}-\left(L^{(1)} S\right)_{\mu \nu}\right], \mu, v=x, y,
\end{aligned}
$$

where

$$
L_{\mu \nu}^{(\alpha)}=\int d E\left(-\frac{\partial f(E)}{\partial E}\right)\left(E-\mu^{*}\right)^{\alpha} \sigma_{\mu \nu}(E),
$$


and $\sigma_{\mu \nu}$ is the zero-temperature energy-dependent conductivity. Note that $\sigma_{\mu \nu}=L_{\mu \nu}^{(0)}$. These expressions have the same form as the standard textbook expressions. Both are valid for independent electrons and elastic scattering. The differences are that the former are fully quantum mechanical and valid for weak electric fields whereas the latter are valid in $k$ space only, but for arbi- trary electric fields.

The finite temperature conductivity components $\sigma_{\mu \nu}$ have been evaluated in the preceding section for scattering by randomly distributed impurities. When the zerotemperature limit of these conductivities, which enters the expressions for $L_{\mu \nu}^{(\alpha)}$, is evaluated to order $V_{0}^{2}$ we obtain

$$
\begin{aligned}
& \left.\left.L_{y y}^{(\alpha)}\right|_{\mathrm{dif}}=2 \frac{e^{2}}{h} \frac{2 \pi^{2}}{\hbar} \frac{l^{2}}{a^{2}} \tau V_{0}^{2} e^{-u} \sum_{n=0}^{\infty}\left[L_{n}(u)\right]^{2}\left(E_{n}-\mu^{*}\right)^{\alpha} \mid-\frac{\partial f(E)}{\partial E}\right]_{E=E_{n}}, \\
& \left.L_{x x}^{(\alpha)}\right|_{\mathrm{col}}=\frac{e^{2}}{h} \frac{N_{I} U_{0}^{2}}{\pi \Gamma_{a}} \sum_{n=0}^{\infty}\left[(2 n+1) I_{n}^{(\alpha)}+\Delta_{n}^{(\alpha)}\right], \\
& L_{y x}^{(\alpha)}=\frac{2 e^{2}}{h} \frac{l^{2}}{a} \sum_{n=0}^{\infty}(n+1) \int_{0}^{a / l^{2}} d k_{y} \frac{1}{\left[\left(E_{n+1, k_{y}}-E_{n, k_{y}}\right) / \hbar \omega_{c}\right]^{2}} \int_{E_{n, k_{y}}}^{E_{n+1, k_{y}}} d E\left(E-\mu^{*}\right)^{\alpha}\left(-\frac{\partial F(E)}{\partial E}\right)_{E=E_{n, k_{y}}} ;
\end{aligned}
$$

here we introduced the notation

$$
\begin{aligned}
& I_{n}^{(\alpha)}=\int_{0}^{a / l^{2}} d k_{y}\left(E_{n, k_{y}}-\mu^{*}\right)^{\alpha}\left(-\frac{\partial f(E)}{\partial E}\right)_{E=E_{n, k_{y}}}, \\
& \Delta_{n}^{(\alpha)}=2\left(\frac{V_{0}}{\hbar \omega_{c}}\right)^{2} u e^{-u}\left\{\left[L_{n-1}^{1}(u)\right]^{2}+\left[L_{n}^{1}(u)\right]^{2}\right. \\
& \left.+L_{n-1}^{1}(u) L_{n}^{1}(u)\right\} J_{n}^{(\alpha)},
\end{aligned}
$$

where

$$
\begin{aligned}
J_{n}^{(\alpha)}=\int_{0}^{a / l^{2}} d k_{y} \sin ^{2}\left(K x_{0}\right)\left(E_{n, k_{y}}-\mu^{*}\right)^{\alpha} \\
\\
\times\left[-\frac{\partial f(E)}{\partial E}\right)_{E=E_{n, k_{y}}} .
\end{aligned}
$$

The final result is $L_{y y}^{(\alpha)}=\left.L_{y y}^{(\alpha)}\right|_{\text {dif }}+\left.L_{y y}^{(\alpha)}\right|_{\text {col }}$ and $L_{x x}^{(\alpha)}=\left.L_{x x}^{(\alpha)}\right|_{\mathrm{col}}$ with $\left.L_{y y}^{(\alpha)}\right|_{\mathrm{col}}=\left.L_{x x}^{(\alpha)}\right|_{\mathrm{col}}$.

In our numerical calculation we consider the same experimental parameters as in the preceding section. The components of the thermopower tensor divided by the temperature are shown in Fig. 10(a) as a function of the magnetic field in units of $-k_{B} / e K . S_{x x}$ and $S_{y y}$ are almost identical and therefore only $S_{x x}$ is shown. Contrary to the conductivity no asymmetry in the thermopower tensor is predicted. The oscillations in the $T=2 \mathrm{~K}$ curves for $B>0.3 \mathrm{~T}$ are $\mathrm{SdH}$ oscillations which have died out when $T=6 \mathrm{~K}$. The modulation contribution to the thermopower, $\Delta S_{\mu \nu}=S_{\mu \nu}\left(V_{0}\right)-S_{\mu \nu}(0)$, is shown in Fig. 10(b). The different tensor components are offset; the zero point is indicated by the position of the horizontal arrows. The amplitude of the oscillations in $\Delta S_{x y}$ is larger than that of the diagonal components $S_{\mu \mu}$. For $B>0.3 \mathrm{~T}$ the Weiss oscillations appear as an envelope to the SdH oscillations. These $\mathrm{SdH}$ oscillations are shifted by $90^{\circ}$ with respect to those of $\Delta S_{x x}$ and $\Delta S_{y y}$.

The different components of the thermal-conductivity tensor are shown in Fig. 11(a) and have a magnetic-field dependence similar to that of the electrical conductivity [see Fig. 6(a)]. This is even more apparent in Fig. 11(b) where we plotted the modulation contribution to the thermal conductivity. These results are very similar to the one corresponding to the electrical conductivity [see Fig. 6(b)]. For higher magnetic fields [see Fig. 11(c)] we observe that the $\mathrm{SdH}$ oscillations have the following
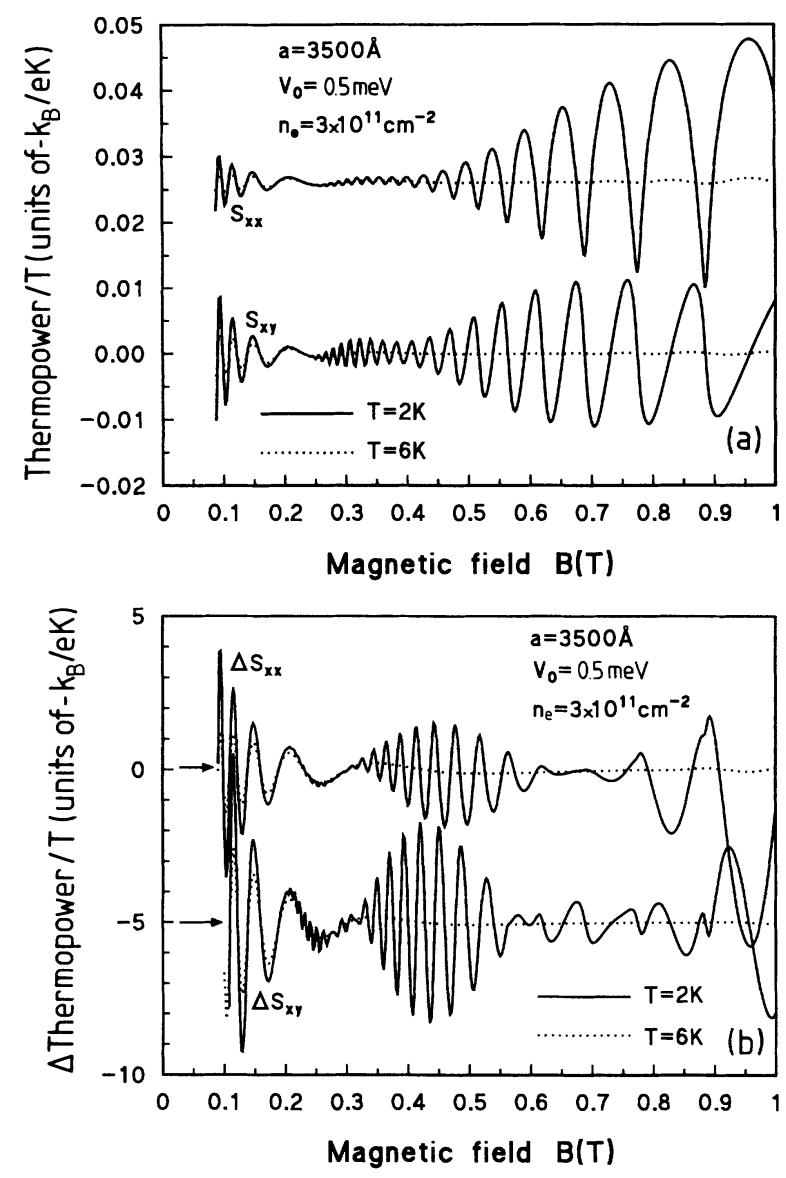

FIG. 10. (a) The thermopower divided by temperature as function of the magnetic field (only $S_{x x}$ is shown because $S_{y y} \approx S_{x x}$ ). (b) The contribution due to the 1D modulation; the different curves are shifted vertically and the zero position is indicated by the arrows, for $T=2 \mathrm{~K}$ (solid curves) and $T=6 \mathrm{~K}$ (dotted curves). 



FIG. 11. The same as Fig. 10 but now for the thermal conductivity.

characteristics: (1) in $K_{y y}$ they are much larger than in $K_{x x}$ similar to the conductivity result; (2) $K_{x y}$ and $\sigma_{x y}=-\sigma_{y x}$ have a very similar magnetic-field dependence; (3) $K_{x x}$ has a maximum where $K_{y y}$ is minimal which is different from the electrical conductivity; (4) the low-temperature SdH peaks in $K_{\mu \mu}$ are very different from those of $\sigma_{\mu \mu}$; and (5) at high temperature $K_{y y}$ and $\sigma_{y y}$ exhibit $\mathrm{SdH}$ oscillations which are $180^{\circ}$ out of phase.

The similarity between the Weiss oscillations in the thermal coefficients and those in the electrical coefficients becomes more apparent if we plot [see Fig. 12(a)] the inverse of the thermal-conductivity tensor which we will call the thermal resistance: $\kappa^{-1}$. Notice the similarity (see Fig. 7) between $\kappa_{x x}^{-1}$ and $\rho_{x x}, \kappa_{y y}^{-1}$ and $\rho_{y y}$, and between $\kappa_{x y}^{-1}$ and $\rho_{x y}$. We have multiplied the thermal-resistance components with temperature in order that the results for $T=2 \mathrm{~K}$ and at $T=6 \mathrm{~K}$ appear in the same figure. Notice that (1) the Weiss oscillations in the thermal resistance are in phase with those of the electrical resistance; (2) they have a stronger temperature dependence than those in the electrical resistance; and (3) the SdH oscilla-

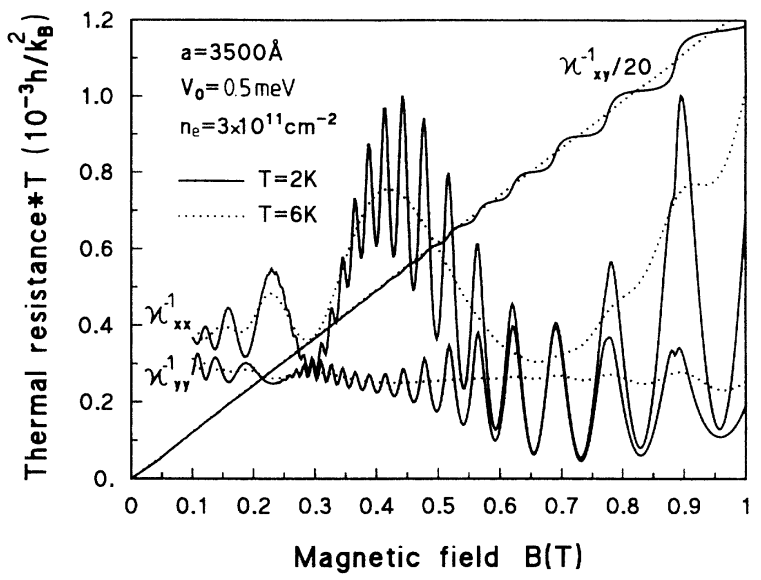

FIG. 12. The components of the thermal-resistance tensor multiplied with temperature as function of the magnetic field, for the two temperatures $T=2 \mathrm{~K}$ (solid curves) and $T=6 \mathrm{~K}$ (dotted curves).

tions are out of phase as compared to those in the electrical resistance.

Measurements of the thermopower of a 2DEG in the absence of a periodic potential have been performed in, e.g., Ref. 32. The above impurity contributions can be observed experimentally by the use of the hot-electron technique, ${ }^{33}$ which relies on the use of gradients in the electron temperature as produced by electron heating instead of the conventional gradients in the lattice temperature. This technique avoids phonon-drag effects and was recently successfully employed to measure the thermopower of a mesoscopic conductor ${ }^{33}$ and of quantum point contacts. $^{34}$

\section{MAGNETO-OPTICS}

The average power absorbed from a circularly polarized light of microwave frequency $\omega$ and electric-field strength $E$ is given by ${ }^{35}$

$$
\begin{aligned}
P(\omega) & =\frac{1}{2} E^{2} \sigma_{ \pm}(\omega) \\
& =\frac{1}{2} E^{2} \operatorname{Re}\left[\sigma_{x x}(\omega)+\sigma_{y y}(\omega)-i \sigma_{x y}(\omega)+i \sigma_{y x}(\omega)\right],
\end{aligned}
$$

where the frequency-dependent conductivity tensor consists of two parts, $\sigma_{\mu \nu}(\omega)=\sigma_{\mu \nu}^{d}(\omega)+\sigma_{\mu \nu}^{n d}(\omega)$. The component $\sigma_{\mu \nu}^{d}(\omega)$ is given by Eqs. (2.55) and (2.64) of Ref. 22. For $w_{c} \tau \gg 1$, which is the experimental situation, it can be shown that $\sigma_{\mu \nu}^{d}(\omega) \ll \sigma_{\mu \nu}^{n d}(\omega)$ for the diffusion contribution (only $\sigma_{y y}$ since $\sigma_{x x}=0$ ) and the collisional one $\sigma_{x x} \approx \sigma_{y y}$. It can also be shown that $\sigma_{y x}^{d}(\omega)$ $\left[\stackrel{x x}{=}-\sigma_{x y}^{d y}(\omega)\right]$ vanishes identically. Therefore, in Eq. (46) we can take $\sigma_{\mu \nu}(\omega) \approx \sigma_{\mu \nu}^{n d}(\omega)$ and evaluate the latter [see Eq. (12)] using Eqs. (17b) and (17c) for the velocity matrix elements

$\sigma_{y x}(\omega) \approx \frac{e^{2} \hbar \omega_{c}}{2 m^{*} A_{0}} \sum_{n, k_{y}}(n+1) \frac{f_{n, k_{y}}-f_{n+1, k_{y}}}{\Delta_{n+1, n, k_{y}}}\left(\frac{\Delta_{n+1, n, k_{y}}+\hbar \omega}{\left(\Delta_{n+1, n, k_{y}}+\hbar \omega\right)^{2}+\Gamma_{n+1, n, k_{y}}^{2}}+\frac{\Delta_{n+1, n, k_{y}}-\hbar \omega}{\left(\Delta_{n+1, n, k_{y}}-\hbar \omega\right)^{2}+\Gamma_{n+1, n, k_{y}}^{2}}\right)$, 
where $\Delta_{n+1, n k_{y}}=\hbar \omega_{c}+V_{0} e^{-u / 2} L_{n+1}^{-1}(u) \cos \left(K x_{0}\right)$ and a collisional broadening was introduced with zero shift of the energy levels. If we assume $\Gamma_{n, n+1, k_{y}} \approx \Gamma_{n, k_{y}}$ we can estimate $\Gamma_{n, k_{y}}$ from $\Gamma_{n, k_{y}} \approx \hbar / \tau \approx\left(N_{I} U_{0}^{2} \pi l^{2}\right)^{1 / 2}$. The result for the corresponding term in $\sigma_{x x}^{n d}(\omega)$ is given by Eq. (47) multiplied by $-i$ and the change $\Delta_{n+1, n k_{y}}-\hbar \omega$ $\rightarrow \hbar \omega-\Delta_{n+1, n k_{y}}$. Combining these results with Eq. (46) we obtain

$$
\begin{aligned}
P(\omega)=\frac{e^{2} \hbar^{2} \omega_{c} E^{2}}{2 m^{*} A_{0}} \sum_{n, k_{y}} & (n+1) \frac{f_{n, k_{y}}-f_{n+1, k_{y}}}{\Delta_{n+1, n, k_{y}}} \\
& \times \frac{\Gamma_{n, k_{y}}}{\left(\Delta_{n+1, n, k_{y}}-\hbar \omega\right)^{2}+\Gamma_{n, k_{y}}^{2}} .
\end{aligned}
$$

In the absence of modulation $\Delta_{n+1, n, k_{y}}=\hbar \omega_{c}$, $\Gamma_{n, k_{y}}=\Gamma_{n}$, the sum over $k_{y}$ gives a factor $A_{0} \pi l^{2}$, and Eq. (48) becomes identical to the well-known expression (3.36) of Ref. 36. We notice in passing that the above derivation misses the so-called "quantum" oscillation ${ }^{37}$ [due to neglect of $\sigma_{y y}^{d}(\omega)$ ] but it is also valid in three dimensions with the changes $A_{0} \rightarrow \Omega_{0}, \Delta_{n+1, n, k_{y}} \rightarrow \hbar \omega_{c}$ (in
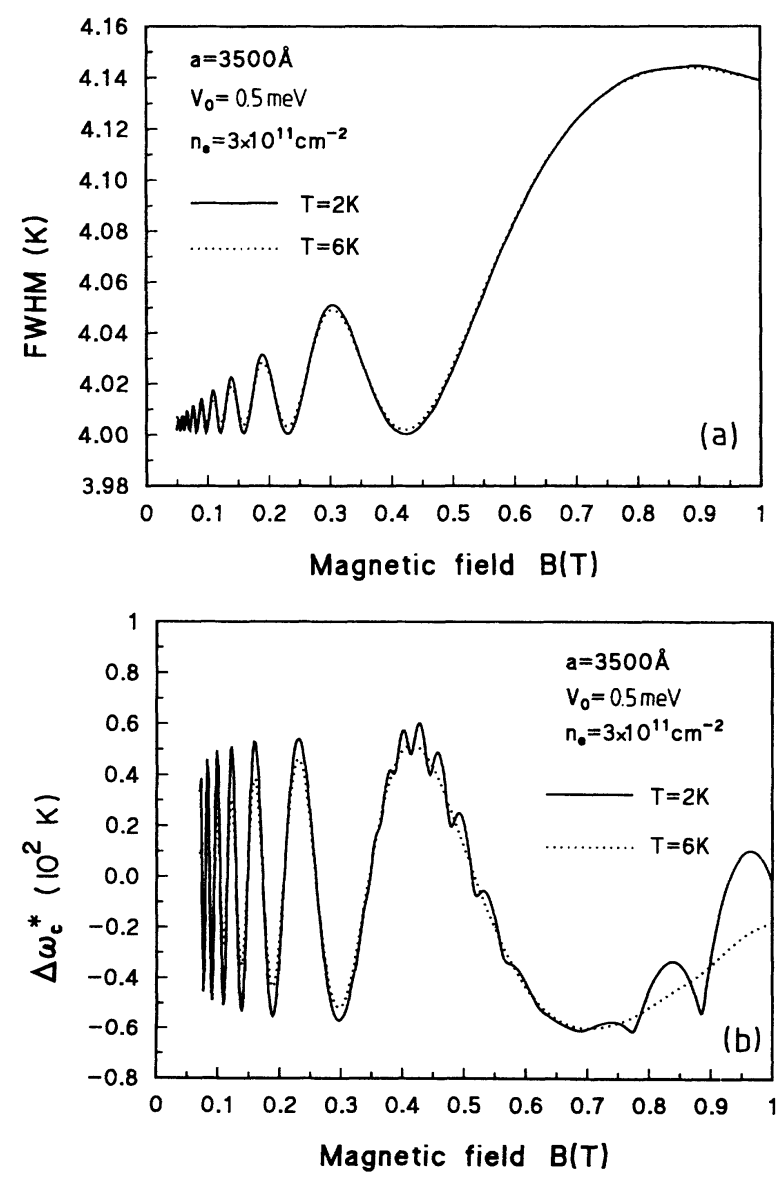

FIG. 13. The shift in the position of the cyclotron resonance line (a) and the linewidth (b) as function of the magnetic field for two different temperatures. We took the broadening $\Gamma=2 \mathrm{~K}$. the absence of modulation), and $\Sigma_{k_{y}} \rightarrow \Sigma_{k_{y} k_{z}}$. When the sum over $k_{y}$ is replaced by an integral, Eq. (48) becomes identical to Eq. (11) of Ref. 10.

The shift of the cyclotron resonance peak position due to the 1D modulation is shown in Fig. 13(b) for $V_{0}=0.5$ meV. The corresponding linewidth (FWHM stands for "full width at half maximum") is depicted in Fig. 13(a). We see that (1) the cyclotron peak shift oscillates in phase with $\rho_{x x}$ and $\rho_{x y} ;(2)$ the linewidth oscillates in phase with $\rho_{y y}$ and is consequently $180^{\circ}$ out of phase with the shift in the cyclotron peak position; and (3) only the peak position exhibits SdH oscillations at higher magnetic fields.

\section{CONCLUSION AND DISCUSSION}

We have performed a detailed investigation of the electrical and thermal properties of a two-dimensional electron gas which is modulated by a weak one-dimensional periodic potential. Such a periodic potential broadens the Landau levels into bands, the width of which is an oscillating function of the magnetic field. This leads to an extra conduction mechanism where electrons are channeled along the 1D potential and they can move through the system by diffusive scattering on impurities. The strength of this band conduction is proportional to the square of the bandwidth of the Landau level at the Fermi energy and thus oscillates as function of the magnetic field. The position of the maxima and minima in the resistivity are determined by a commensurability condition between the period $a$ of the 1D potential and the diameter of the cyclotron orbit $2 R_{c}$. The resistivity perpendicular $\left(\rho_{x x}\right)$ to the 1D periodic potential shows pronounced

TABLE I. Characteristics of the Weiss oscillations in a 2DEG of density $3 \times 10^{11} \mathrm{~cm}^{-2}$ and mobility $\mu=10^{6} \mathrm{~cm}^{2} / \mathrm{V} \mathrm{s}$ for a $1 \mathrm{D}$ potential modulation of strength $V_{0}=0.5 \mathrm{meV}$ and period $a=3500 \AA$. The percentage magnitude of the amplitude of the Weiss oscillations of the different physical quantities is taken around $B=0.2-0.3 \mathrm{~T}$. The phase shift of the oscillations is referred to the one of the resistivity $\rho_{x x}$.

\begin{tabular}{lcrr}
\hline & & \multicolumn{2}{c}{ Phase shift } \\
\cline { 4 - 5 } Quantity & Amplitude $(\%)$ & Weiss & SdH \\
\hline Resistivity & & & \\
$\rho_{x x}$ & 50 & $0^{\circ}$ & $0^{\circ}$ \\
$\rho_{y y}$ & 20 & $180^{\circ}$ & $0^{\circ}$ \\
$\rho_{x y}$ & 0.6 & $0^{\circ}$ & $90^{\circ}$ \\
$d R_{H} / d B$ & 15 & $90^{\circ}$ & $180^{\circ}$ \\
& & & \\
Thermopower & 10 & & \\
$S_{x x} \approx S_{y y}$ & 100 & $90^{\circ}$ & $0^{\circ}$ \\
$S_{x y}$ & & $90^{\circ}$ & $90^{\circ}$ \\
& & & \\
Thermal resistance & 50 & & \\
$A_{x x}$ & 20 & $180^{\circ}$ & $180^{\circ}$ \\
$A_{y y}$ & 35 & $0^{\circ}$ & $90^{\circ}$ \\
$A_{x y}$ & & & \\
Cyclotron resonance & & $0^{\circ}$ & $0^{\circ}$ \\
position & 0.2 & $180^{\circ}$ & \\
FWHM & 1.5 & & \\
\hline \hline
\end{tabular}


oscillations with period $\sim \sqrt{n_{e}} / B$, where an increase of the resistivity by a factor of more than 2 is easily attainable. Weaker oscillations in the resistivity component parallel to the 1D potential are observed which exhibit minima when $\rho_{x x}$ is maximum. The reason behind this difference is that the resistivity $\rho_{y y}$ is determined by the value of the DOS squared at the Fermi energy, while $\rho_{x x}$ is mainly determined by the width of the Landau level at $E_{F}$. Similar Weiss oscillations were found in (1) the Hall resistance; (2) the thermodynamic quantities like the magnetization, the susceptibility, and the specific heat; (3) the thermal transport coefficients like the thermopower, the thermal conductivity and the thermal resistivity; and (4) the position and width of the cyclotron resonance line. The magnitude and the phase of the Weiss oscillations of the different physical quantities are summarized in Table I. The percentage magnitude of the amplitude of the os- cillation of the quantity $X$ is defined as $100[X(\max )-X(\min )] / X(\max )$ where the values of $X$ are taken at the extrema near $B \sim 0.2-0.3 \mathrm{~T}$ with the minima taken at a lower $B$ value. For the present situation of weak modulation strength, the amplitude of the Weiss oscillations of the different physical quantities is proportional to $V_{0}^{2}$. The phase of the oscillation of the different quantities is compared to the one of $\rho_{x x}$. In Table I we also give the phase of the $\mathrm{SdH}$ oscillations.

\section{ACKNOWLEDGMENTS}

This work was supported by NSERC Grant No. URF0093498 and by the Collaborative Research Grant NATO 5-2-05/RG No. 890123. One of us (F.M.P.) is supported by the Belgian National Science Foundation.
${ }^{1}$ D. Weiss, K. von Klitzing, K. Ploog, and G. Weimann, Europhys. Lett. 8, 179 (1989); and in High Magnetic Fields in Semiconductor Physics II, edited by G. Landwehr (SpringerVerlag, Wurtzburg, 1989), p. 357.

${ }^{2}$ R. R. Gerhardts, D. Weiss, and K. von Klitzing, Phys. Rev. Lett. 62, 1173 (1989).

${ }^{3}$ R. W. Winkler, J. P. Kotthaus, and K. Ploog, Phys. Rev. Lett. 62, 1177 (1989).

${ }^{4}$ E. S. Alves, P. H. Beton, M. Henini, L. Eaves, P. C. Main, O. H. Hughes, G. A. Toombs, S. P. Beaumont, and C. D. W. Wilkinson, J. Phys. Condens. Matter 1, 8257 (1989).

${ }^{5}$ D. Weiss, K. von Klitzing, K. Ploog, and G. Weimann, Surf. Sci. 229, 88 (1990); D. Weiss (unpublished); R. R. Gerhardts, D. Weiss, and U. Wulf, Phys. Rev. B 43, 5192 (1991).

${ }^{6}$ R. A. Puechner, J. Ma, R. Mezenner, W.-P. Liu, A. M. Kriman, G. N. Maracas, G. Bernstein, D. K. Ferry, P. Chu, H. H. Wieder, and P. Newman, Surf. Sci. 228, 520 (1990); K. Ismail, D. A. Antoniadis, H. I. Smith, C. T. Liu, K. Nakamura, and D. C. Tsui, J. Vac. Sci. Technol. B 7, 2000 (1989); K. Ismail, T. P. Smith III, W. T. Masselink, and H. I. Smith, Appl. Phys. Lett. 55, 2766 (1989); A. Toriumi, K. Ismail, M. Burkhardt, D. A. Antoniadis, and H. I. Smith, Phys. Rev. B 41, 12346 (1990).

${ }^{7}$ H. Fang and P. J. Stiles, Phys. Rev. B 41, 10171 (1990).

${ }^{8}$ D. R. Hofstadter, Phys. Rev. B 14, 2239 (1979).

${ }^{9}$ C. W. J. Beenakker, Phys. Rev. Lett. 62, 2020 (1989).

${ }^{10}$ P. Vasilopoulos and F. M. Peeters, Phys. Rev. Lett. 63, 2120 (1989).

${ }^{11}$ C. Zhang and R. R. Gerhardts, Phys. Rev. B 41, 12850 (1990).

${ }_{12}$ P. Středa and A. H. MacDonald, Phys. Rev. B 41, 11892 (1990).

${ }^{13}$ F. M. Peeters and P. Vasilopoulos, Phys. Rev. B 42, 5899 (1990).

${ }^{14}$ P. G. Harper, Proc. Phys. Soc. London, Sect. A 68, 874 (1955); A 68, 879 (1955); G. E. Sil'berman, Zh. Eksp. Teor. Fiz. 32, 296 (1957) [Sov. Phys. JETP 5, 208 (1957)] 33, 387 (1957); [6, 299 (1958)].

${ }^{15}$ H. Shiba and H. Fukuyama, J. Phys. Soc. Jpn. 26, 910 (1969).

${ }^{16}$ A. M. Berezhkovskîi and R. A. Suris, Zh. Eksp. Teor. Fiz. 86, 193 (1984) [Sov. Phys. JETP 59, 109 (1984)]; A. V. Chaplik, Solid State Commun. 53, 539 (1985).

${ }^{17}$ J.-K. Maan, in Festkörperprobleme (Advances in Solid State Physics), edited by P. Grosse (Pergamon, Braunschweig,
1987), Vol. 27, p. 137.

${ }^{18}$ G. R. Aizin and V. A Volkov, Zh. Eksp. Teor. Fiz. 87, 1469 (1984) [Sov. Phys. JETP 60, 844 (1984)].

${ }^{19}$ C. T. Cui, V. Fessatidis, and N. J. M. Horing, Phys. Rev. Lett. 63, 2598 (1989).

${ }^{20}$ D. Weiss, C. Zhang, R. R. Gerhardts, K. von Klitzing, and G. Weimann, Phys. Rev. B 39, 13020 (1989).

${ }^{21}$ W. Zawadzki and R. Lassnig, Surf. Sci. 142, 225 (1984).

${ }^{22}$ R. Abe, Statistical Mechanics (University of Tokyo Press, Tokyo, 1975), p. 28.

${ }^{23}$ M. Charbonneau, K. M. Van Vliet, and P. Vasilopoulos, J. Math. Phys. 23, 318 (1982).

${ }^{24}$ P. Vasilopoulos and C. M. Van Vliet, J. Math. Phys. 25, 1391 (1984); Phys. Rev. B 34, 1057 (1986); 34, 4375 (1986).

${ }^{25}$ E. N. Adams and T. D. Holstein, J. Phys. Chem. Solids 10, 254 (1959); P. N. Argyres and L. M. Roth, ibid. 12, 89 (1959); R. Kubo, S. J. Miyake, and N. Hashitsume, Solid State Phys. 17, 269 (1965).

${ }^{26}$ P. Vasilopoulos, Phys. Rev. B 32, 771 (1985).

${ }^{27}$ See, e.g., T. Ando, A. B. Fowler, and F. Stern, Rev. Mod. Phys. 54, 437 (1982).

${ }^{28}$ R. J. Haug, R. R. Gerhardts, K. von Klitzing, and K. Ploog, Phys. Rev. Lett. 59, 1349 (1987).

${ }^{29}$ M. Davison, E. S. Alves, M. Dellow, P. H. Beton, R. P. Taylor, P. C. Main, L. Eaves, S. P. Beaumont, and C. D. W. Wilkinson, in Proceedings of the 20th International Conference on the Physics of Semiconductors, edited by E. M. Anastassakis and J. D. Joannopoulos (World Scientific, Singapore, 1990), p. 2423.

30J. P. Harrang, R. J. Higgins, R. K. Goodall, P. R. Jay, M. Laviron, and P. Delescluse, Phys. Rev. B 32, 8126 (1985); S. Das Sarma and F. Stern, ibid. 32, 8442 (1985).

${ }^{31}$ L. Smrčka and P. Středa, J. Phys. C 10, 2153 (1977).

${ }^{32}$ See, e.g., H. Oblok, K. von Klitzing, K. Ploog, and G. Weimann, Surf. Sci. 170, 292 (1986), and references therein.

${ }^{33}$ B. L. Gallagher, T. Galloway, P. H. Beton, J. P. Oxley, S. P. Beaumont, S. Thoms, and C. D. W. Wilkinson, Phys. Rev. Lett. 64, 2058 (1990).

${ }^{34}$ L. W. Molenkamp. H. van Houten, C. W. J. Beenakker, R. Eppenga, and C. T. Foxon, Phys. Rev. Lett. 65, 1052 (1990).

${ }^{35}$ A. Lodder and S. Fujita, J. Phys. Soc. Jpn. 25, 774 (1968).

${ }^{36}$ M. Prasad and S. Fujita, Physica 91A, 1 (1978).

${ }^{37}$ T. Ando, J. Phys. Soc. Jpn. 38, 989 (1975). 